\title{
A STUDY ON NEW RIGHT/LEFT INVERSES OF NONSQUARE POLYNOMIAL MATRICES
}

\author{
WoJcIECH P. HUNEK, KRZYSZTOF J. LATAWIEC \\ Institute of Control and Computer Engineering \\ Opole University of Technology, ul. Sosnkowskiego 31, 45-272 Opole, Poland \\ e-mail: \{w.hunek, k. latawiec\}@po.opole.pl
}

\begin{abstract}
This paper presents several new results on the inversion of full normal rank nonsquare polynomial matrices. New analytical right/left inverses of polynomial matrices are introduced, including the so-called $\tau$-inverses, $\sigma$-inverses and, in particular, $S$-inverses, the latter providing the most general tool for the design of various polynomial matrix inverses. The applicationoriented problem of selecting stable inverses is also solved. Applications in inverse-model control, in particular robust minimum variance control, are exploited, and possible applications in signal transmission/recovery in various types of MIMO channels are indicated.
\end{abstract}

Keywords: multivariable systems, right/left inverses of polynomial matrices, Smith factorization, minimum variance control.

\section{Introduction}

Whilst the task of the Moore-Penrose inversion of polynomial matrices (or rational matrices) has attracted considerable research interest (Ben-Israel and Greville, 2003; Karampetakis and Tzekis, 2001; Kon'kova and Kublanovskaya, 1996; Stanimirović, 2003; Stanimirović and Petković, 2006; Varga, 2001; Vologiannidis and Karampetakis, 2004; Zhang, 1989), the problem of right/left inverting nonsquare (full normal rank) polynomial matrices has not been given proper attention by the academia. The suggested control applications (Bańka and Dworak, 2006; 2007; Chen and Hara, 2001; Ferreira, 1988; Hautus and Heymann, 1983; Quadrat, 2004; Trentelman et al., 2001; Williams and Antsaklis, 1996) have not ended up with algorithms for obtaining right/left polynomial matrix inverses and their quantification. One possible reason could be an infinite number of solutions to the problem, the ambiguity impeding the general analytical outcome on the one hand and raising confusion with the selection of a 'proper' inverse on the other.

A common way out, not to say getting around the problem, has been the employment of the familiar minimum-norm right or least-squares left inverses. Those unique inverses are in fact 'optimal' in some sense, so under the lack of any 'competitive' inverses they could be thought of as the best ones. Such a minimum- norm/least-squares solution has been encountered in applications of right/left inverses of nonsquare polynomial matrices or nonsquare rational matrices, to mention control analysis and design problems (Kaczorek, 2005; Kaczorek et al., 2009; Latawiec, 1998; Williams and Antsaklis, 1996) as well as error-control coding (Fornasini and Pinto, 2004; Forney, 1991; Gluesing-Luerssen et al., 2006; Johannesson and Zigangirov, 1999; Lin and Costello, 2004; Moon, 2005) and perfect reconstruction filter banks (Bernardini and Rinaldo, 2006; Gan and Ling, 2008; Quevedo et al., 2009).

The employment of the minimum-norm right or least-squares left inverses has also been the authors' first choice when solving the problem of the generation of the so-called 'control zeros' for a nonsquare LTI MIMO system, the zeros defined as poles of an inverse system or poles of a closed-loop Minimum Variance Control (MVC) system (Latawiec, 1998; Latawiec et al., 2000). The limited usefulness of minimum-norm right or least-squares left inverses, to be in the sequel called $T$-inverses, has soon brought us to the point where we have introduced the so-called $\tau$-inverses and $\sigma$-inverses of nonsquare polynomial matrices (Hunek, 2008; Latawiec, 2004; Latawiec et al., 2004; 2005).

Since in some applications it is welcome for an inverse polynomial matrix not to have any pole at all, we have offered pole-free right/left inverses of nonsquare polyno- 
mial matrices (Hunek, 2008; 2009a; 2009b; Hunek et al., 2007 ; 2010). At last, we have presented a specific Smith factorization solution to the inverse problem for nonsquare polynomial matrices (Hunek, 2008; 2009a; Hunek et al., 2007).

In this paper, the Smith factorization approach is extended to finally obtain a new general class of inverses, valid for any nonsquare polynomial matrices and providing an arbitrary number of degrees of freedom in terms of a preselected number (and value) of the inverse's zeros and poles, if any. For completeness, this paper recalls all the above mentioned (and presented mainly at conferences) new results in the inversion of nonsquare polynomial matrices. Applications of the results in process control technology have been reported (Hunek, 2008; 2009a; Hunek and Latawiec, 2009; Hunek et al., 2007; Latawiec, 2004; 2005; Latawiec et al., 2004; 2005) and are now being expanded (Hunek et al., 2010), whilst possible applications in, e.g., error coding control and perfect reconstruction filter banks seem forthcoming.

This paper is structured as follows. Having introduced the inversion problem for full normal rank polynomial matrices, system representations including the polynomial matrices to be inverted are reviewed in Section 2. Since our new concepts of polynomial matrix inversion have originated from closed-loop discrete-time MVC, this control strategy is recalled in Section 3. A fundamental idea behind the forthcoming introduction of new, MVC-related inverses of nonsquare polynomial matrices is illustrated in the instructive motivating example of Section 4. Analytical expressions for new polynomial matrix inverses, including the so-called $\tau$-inverses and $\sigma$-inverses, in addition to the well-known but renamed $T$-inverse, are offered in Section 5. Control-related applications call for the selection of stable polynomial matrix inverses, which is covered in Sections 6 and 7. The Smith factorization approach of the latter is extended in Section 8 to culminate in the introduction of a new, general $S$-inverse of a nonsquare polynomial matrix. Actual and potential applications are indicated in Section 9. The discussion in Section 10 provides yet another justification for setting the inversion problem in the time-domain framework. Also, a series of open research problems are specified in that section. New results of the paper are summarized in the conclusions of Section 11.

\section{System representations}

We start from general system representations related to control applications of inverse polynomial matrices, including process control, error-control coding and perfect reconstruction filter banks.

Consider an $n_{u}$-input $n_{y}$-output Linear TimeInvariant (LTI) discrete-time system with the input $u(t)$ and the output $y(t)$, described by possibly rectangular transfer-function matrix $G \in \mathbb{R}^{n_{y} \times n_{u}}(z)$ in the complex operator $z$. The transfer function matrix can be represented in the Matrix Fraction Description (MFD) form $G(z)=A^{-1}(z) B(z)$, where the left coprime polynomial matrices $A \in \mathbb{R}^{n_{y} \times n_{y}}[z]$ and $B \in \mathbb{R}^{n_{y} \times n_{u}}[z]$ can be given in the form $A(z)=z^{n} I_{n_{y}}+\cdots+a_{n}$ and $B(z)=z^{m} b_{0}+\cdots+b_{m}$, respectively, where $n$ and $m$ are the orders of the respective matrix polynomials and $I_{n_{y}}$ is the identity $n_{y}$-matrix. An alternative MFD form $G(z)=$ $\tilde{B}(z) \tilde{A}^{-1}(z)$, involving right coprime $\tilde{A} \in \mathbb{R}^{n_{u} \times n_{u}}[z]$ and $\tilde{B} \in \mathbb{R}^{n_{y} \times n_{u}}[z]$, can also be tractable here but in a less convenient way (Latawiec, 1998).

Algorithms for the calculation of MFDs are known and software packages in Matlab's Polynomial Toolbox ${ }^{\circledR}$ are available. Unless necessary, we will not discriminate between $\underline{A}\left(z^{-1}\right)=I_{n_{y}}+\cdots+\underline{a}_{n} z^{-n}$ and $A(z)=$ $z^{n} \underline{A}\left(z^{-1}\right)$, nor between $\underline{B}\left(z^{-1}\right)=\underline{b}_{0}+\cdots+\underline{b}_{m} z^{-m}$ and $B(z)=z^{m} \underline{B}\left(z^{-1}\right)$ with $G(z)=A^{-1}(z) \underline{B}(z)=$ $z^{-d} \underline{A}^{-1}\left(z^{-1}\right) \underline{B}\left(z^{-1}\right)$, where $d=n-m$ is the time delay of the system. In the sequel, we will assume for clarity that $B(z)$ is of full normal rank; a more general case of $B(z)$ being of non-full normal rank can also be tractable (Latawiec, 1998).

Let us finally concentrate on the case when the normal rank of $B(z)$ is $n_{y}$ ('symmetrical' considerations can be made for the normal rank $n_{u}$ ). The first MFD form can be directly obtained from the AR(I)X/AR(I)MAX model of a system $\underline{A}\left(q^{-1}\right) y(t)=q^{-d} \underline{B}\left(q^{-1}\right) u(t)+$ $\left[\underline{C}\left(q^{-1}\right) / \underline{D}\left(q^{-1}\right)\right] v(t)$, where $q^{-1}$ is the backward shift operator and $v(t) \in \mathbb{R}^{n_{y}}$ is the uncorrelated zero-mean disturbance at (discrete) time $t$. The pairs $A$ and $B$ as well as $A$ and $C \in \mathbb{R}^{n_{y} \times n_{y}}[z]$ are relatively prime polynomial matrices, with (stable) $\underline{C}\left(z^{-1}\right)=\underline{c}_{0}+\cdots+\underline{c}_{k} z^{-k}$ and $k \leq n$, and the $\underline{D}$ polynomial in the $z^{-1}$-domain is often equal to $1-z^{-1}$ (or to unity in the discrete-time MVC considerations). In the sequel, we will also use the operator $w=z^{-1}$ (or $w=q^{-1}$, depending on the context), whose correspondence to the $s$ operator for continuoustime systems has been pioneeringly explored by Hunek and Latawiec (2009).

The familiar Smith-McMillan form $S_{M}(w)$ of $G(w)=w^{d} \underline{A}^{-1}(w) \underline{B}(w)$ (as a special case of the MFD factorization (Desoer and Schulman, 1974)) is given by $G(w)=U(w) S_{M}(w) V(w)$, where $U \in \mathbb{R}^{n_{y} \times n_{y}}[w]$ and $V \in \mathbb{R}^{n_{u} \times n_{u}}[w]$ are unimodular and the pencil $S_{M} \in$ $\mathbb{R}^{n_{y} \times n_{u}}(w)$ is of the form

$$
S_{M}(w)=\left[\begin{array}{cc}
M_{r \times r} & 0_{r \times\left(n_{u}-r\right)} \\
0_{\left(n_{y}-r\right) \times r} & 0_{\left(n_{y}-r\right) \times\left(n_{u}-r\right)}
\end{array}\right],
$$

with $M(w)=\operatorname{diag}\left(\varepsilon_{1} / \psi_{1}, \varepsilon_{2} / \psi_{2}, \ldots, \varepsilon_{r} / \psi_{r}\right)$, where $\varepsilon_{i}(w)$ and $\psi_{i}(w), i=1, \ldots, r$ (with $r$ being the normal rank of $G(w)$ ), are monic coprime polynomials such that $\varepsilon_{i}(w)$ divides $\varepsilon_{i+1}(w), i=1, \ldots, r-1$, and $\psi_{i}(w)$ divi$\operatorname{des} \psi_{i-1}(w), i=2, \ldots, r$. In particular, the Smith form 
is given by the appropriate pencil $S(w)$, with $M(w)=$ $\operatorname{diag}\left(\varepsilon_{1}, \varepsilon_{2}, \ldots, \varepsilon_{r}\right)$ often associated with Smith zeros or transmission zeros (Kaczorek et al., 2009; Tokarzewski, 2002; 2004). The polynomials $\varepsilon_{i}(w)$ are often called the invariant factors of $G(w)$ and their product $\varepsilon(w)=$ $\Pi_{1}^{r} \varepsilon_{i}(w)$ is sometimes referred to as the zero polynomial of $G(w)$.

\section{Closed-loop discrete-time minimum variance control}

Our interest in minimum variance control results from the fact that it is a sort of inverse-model control, directly involving an inverse numerator matrix of the MFD system representation.

In the MVC framework, we consider the ARMAX system description

$$
\underline{A}\left(q^{-1}\right) y(t)=q^{-d} \underline{B}\left(q^{-1}\right) u(t)+\underline{C}\left(q^{-1}\right) v(t) .
$$

For general purposes and duality with the continuous-time case, we use here the ARMAX model, even though it is well known that the $\underline{C}\left(q^{-1}\right)$ polynomial matrix of disturbance parameters is usually in control engineering practice unlikely to be effectively estimated (and is often used as a control design, observer polynomial matrix instead).

All the results to follow can be dualized for continuous-time systems described by a Laplace operator model analogous to Eqn. (2). This can be enabled owing to the unified, discrete-time/continuous-time MVC framework introduced by Hunek and Latawiec (2009).

Consider a right-invertible system $\left(n_{y}<n_{u}\right)$ described by Eqn. (2) and assume that the observer (or disturbance-related) polynomial $\underline{C}\left(q^{-1}\right)=\underline{c}_{0}+\underline{c}_{1} q^{-1}+$ $\cdots+\underline{c}_{k} q^{-k}$ has all its roots inside the unit disk.

Then the general MVC law, minimizing the performance index

$$
\begin{array}{r}
\min _{u(t)} E\left\{\left[y(t+d)-y_{\mathrm{ref}}(t+d)\right]^{\mathrm{T}}\right. \\
\left.\left[y(t+d)-y_{\mathrm{ref}}(t+d)\right]\right\},
\end{array}
$$

where $y_{\mathrm{ref}}(t+d)$ and $y(t+d)=\underline{\tilde{C}}^{-1}\left(q^{-1}\right)\left[\underline{\tilde{F}}\left(q^{-1}\right)\right.$. $\left.\underline{B}\left(q^{-1}\right) u(t)+\underline{\tilde{H}}\left(q^{-1}\right) y(t)\right]+\underline{F}\left(q^{-1}\right) v(t)$ are the output reference/setpoint and the stochastic output predictor, respectively, is of the form (Hunek, 2008; Latawiec, 2004)

$$
u(t)=\underline{B}^{\mathrm{R}}\left(q^{-1}\right) \underline{y}(t),
$$

where

$$
\underline{y}(t)=\underline{\tilde{F}}^{-1}\left(q^{-1}\right)\left[\underline{\tilde{C}}\left(q^{-1}\right) y_{\mathrm{ref}}(t+d)-\underline{\tilde{H}}\left(q^{-1}\right) y(t)\right] .
$$

The appropriate polynomial $\left(n_{y} \times n_{y}\right)$-matrices $\underline{\tilde{F}}\left(q^{-1}\right)=I_{n_{y}}+\underline{\tilde{f}}_{1} q^{-1}+\cdots+\underline{\tilde{f}}_{d-1} q^{-d+1}$ and $\underline{\tilde{H}}\left(q^{-1}\right)=$ $\underline{\tilde{h}}_{0}+\underline{\tilde{h}}_{1} q^{-1}+\cdots+\underline{\tilde{h}}_{n-1} q^{-n+1}$ are computed from the polynomial matrix identity (called the Diophantine equation)

$$
\underline{\tilde{C}}\left(q^{-1}\right)=\underline{\tilde{F}}\left(q^{-1}\right) \underline{A}\left(q^{-1}\right)+q^{-d} \underline{\tilde{H}}\left(q^{-1}\right),
$$

with

$$
\underline{\tilde{C}}\left(q^{-1}\right) \underline{F}\left(q^{-1}\right)=\underline{\tilde{F}}\left(q^{-1}\right) \underline{C}\left(q^{-1}\right),
$$

where $\underline{F}\left(q^{-1}\right)=I_{n_{y}}+\underline{f}_{1} q^{-1}+\cdots+\underline{f}_{d-1} q^{-d+1}$, $\underline{\tilde{C}}\left(q^{-1}\right)=\underline{\tilde{c}}_{0}+\underline{\tilde{c}}_{1} q^{-1}+\cdots+\underline{\tilde{c}}_{k} q^{-k}$. For right-invertible systems, the symbol $\underline{B}^{\mathrm{R}}\left(q^{-1}\right)$ denotes, in general, an infinite number of right inverses of the numerator polynomial matrix $\underline{B}\left(q^{-1}\right)$.

Remark 1. The MVC problem reduces to the perfect control one when $v(t)=0$ (with both control laws being identical) and specializes to the perfect regulation problem or to the output (predictor) zeroing one when $y_{\text {ref }}=0$ and $v(t)=0$.

Remark 2. Clearly, the interest in MVC is due to the fact that an inverse polynomial matrix $\underline{B}^{\mathrm{R}}\left(q^{-1}\right)$ is involved here, with poles of the inverse constituting the so-called control zeros. Transmission zeros, if any, make a subset of the set of control zeros (Latawiec, 1998; Latawiec et al., 2000).

Remark 3. The above MVC result and all the results to follow can be dualized for left-invertible systems $\left(n_{y}>\right.$ $n_{u}$ ), with a left inverse of the appropriate matrix involved.

\section{Motivating example}

Consider a multivariable second-order system governed by the ARX model $y(t)+\underline{a}_{1} y(t-1)+\underline{a}_{2} y(t-2)=$ $\underline{b}_{0} u(t-1)+\underline{b}_{1} u(t-2)+\underline{b}_{2} u(t-3)+v(t)$, with the notation as in Section 2. Assume once again that the appropriate polynomial matrix $\underline{B}\left(q^{-1}\right)$ is of full normal rank $n_{y}$ and its (nonunique) right inverse is denoted by $\underline{B}^{\mathrm{R}}\left(q^{-1}\right)$. Equating, in the standard perfect control manner, the (deterministic part of the) one-step output predictor to the reference/setpoint, we obtain

$$
\begin{aligned}
\underline{b}_{0} u(t)+\underline{b}_{1} u(t-1)+\underline{b}_{2} u(t-2)- & \underline{a}_{1} y(t)-\underline{a}_{2} y(t-1) \\
& =y_{\mathrm{ref}}(t+1) .
\end{aligned}
$$

On the one hand, Eqn. (7) immediately leads to the $\mathrm{MV} /$ perfect control law

$$
u(t)=\left(\underline{b}_{0}+\underline{b}_{1} q^{-1}+\underline{b}_{2} q^{-2}\right)^{\mathrm{R}} \underline{y}(t),
$$

with $y(t)=y_{\text {ref }}(t+1)+\underline{a}_{1} y(t)+\underline{a}_{2} y(t-1)$. Equation 8 represents one set of solvers (4) of Eqn. (7) for $u(t)$.

But on the other hand, assuming that $\underline{b}_{0}$ is of full normal rank, Eqn. (7) can be given the form $u(t)=$ 
$\left(\underline{b}_{0}\right)^{\mathrm{R}}\left[\underline{y}(t)-\underline{b}_{1} u(t-1)-\underline{b}_{2} u(t-2)\right]$, which can be rewritten as

$$
u(t)=\left[I_{n_{u}}+\left(\underline{b}_{0}\right)^{\mathrm{R}}\left(\underline{b}_{1} q^{-1}+\underline{b}_{2} q^{-2}\right)\right]^{-1}\left(\underline{b}_{0}\right)^{\mathrm{R}} \underline{y}(t),
$$

representing another set of solvers (4) of Eqn. (7).

Although both MVC laws (8) and (9) are derived from the same output predictor as in Eqn. (7), it is rather surprising that these laws are generally different and this is because $\underline{B}^{\mathrm{R} 1}\left(q^{-1}\right)=\left(\underline{b}_{0}+\underline{b}_{1} q^{-1}+\underline{b}_{2} q^{-2}\right)^{\mathrm{R}} \neq$ $\left[I_{n_{u}}+\left(\underline{b}_{0}\right)^{\mathrm{R}}\left(\underline{b}_{1} q^{-1}+\underline{b}_{2} q^{-2}\right)\right]^{-1}\left(\underline{b}_{0}\right)^{\mathrm{R}}=\underline{B}^{\overline{\mathrm{R}} 2}\left(q^{-1}\right)$, in general. The difference results from specific properties of right inverses for polynomial matrices. Of course, both $\underline{B}\left(q^{-1}\right) \underline{B}^{\mathrm{R} 1}\left(q^{-1}\right)=I_{n_{y}}$ and $\underline{B}\left(q^{-1}\right) \underline{B}^{\mathrm{R} 2}\left(q^{-1}\right)=I_{n_{y}}$.

Observe that a solver to Eqn. (7) can be given yet another form, e.g., $u(t)=\left(\underline{b}_{1} q^{-1}\right)^{\mathrm{R}}\left[y(t)-\underline{b}_{0} u(t)-\right.$ $\left.\underline{b}_{2} u(t-2)\right]$, which can be rewritten as $u(t)=\left[I_{n_{u}}+\right.$ $\left.\left(\underline{b}_{1} q^{-1}\right)^{\mathrm{R}}\left(\underline{b}_{0}+\underline{b}_{2} q^{-2}\right)\right]^{-1}\left(\underline{b}_{1} q^{-1}\right)^{\mathrm{R}} y(t)$ representing another set of solvers (4) of Eqn. (7) for $u(t)$ and giving rise to the introduction of yet another inverse of $\underline{B}\left(q^{-1}\right)$, say $\underline{B}^{\mathrm{R} 3}\left(q^{-1}\right)=\left[I_{n_{u}}+\left(\underline{b}_{1} q^{-1}\right)^{\mathrm{R}}\left(\underline{b}_{0}+\right.\right.$ $\left.\left.\underline{b}_{2} q^{-2}\right)\right]^{-1}\left(\underline{b}_{1} q^{-1}\right)^{\mathrm{R}}$. A similar result can be obtained with the inverse $\underline{B}^{\mathrm{R} 4}\left(q^{-1}\right)=\left[I_{n_{u}}+\left(\underline{b}_{2} q^{-2}\right)^{\mathrm{R}}\left(\underline{b}_{0}+\right.\right.$ $\left.\left.\underline{b}_{1} q^{-1}\right)\right]^{-1}\left(\underline{b}_{2} q^{-2}\right)^{\mathrm{R}}$. But this is not the end. Another form of Eqn. (7) could be $u(t)=\left(\underline{b}_{0}+\underline{b}_{1} q^{-1}\right)^{\mathrm{R}}[\underline{y}(t)-$ $\underline{b}_{2} q^{-2} u(t)$ ], resulting in one more set of solvers $\bar{u}(t)=$ $\left[I_{n_{u}}+\left(\underline{b}_{0}+\underline{b}_{1} q^{-1}\right)^{\mathrm{R}} \underline{b}_{2} q^{-2}\right]^{-1}\left(\underline{b}_{0}+\underline{b}_{1} q^{-1}\right)^{\mathrm{R}} \underline{y}(t)$, with another inverse of $\underline{B}\left(q^{-1}\right)$, say $\underline{B}^{\mathrm{R} 5}\left(q^{-1}\right)=\left[I_{n_{u}}+\left(\underline{b}_{0}+\right.\right.$ $\left.\left.\underline{b}_{1} q^{-1}\right)^{\mathrm{R}} \underline{b}_{2} q^{-2}\right]^{-1}\left(\underline{b}_{0}+\underline{b}_{1} q^{-1}\right)^{\mathrm{R}}$. But, by the same token, we could still introduce another set of solvers related to another inverse, say $\underline{B}^{\mathrm{R} 6}\left(q^{-1}\right)=\left[I_{n_{u}}+\left(\underline{b}_{0}+\right.\right.$ $\left.\left.\underline{b}_{2} q^{-2}\right)^{\mathrm{R}} \underline{b}_{1} q^{-1}\right]^{-1}\left(\underline{b}_{0}+\underline{b}_{2} q^{-2}\right)^{\mathrm{R}}$ and, at last, $\underline{B}^{\mathrm{R} 7}\left(q^{-1}\right)=$ $\left[I_{n_{u}}+\left(\underline{b}_{1} q^{-1}+\underline{b}_{2} q^{-2}\right)^{\mathrm{R}} \underline{b}_{0}\right]^{-1}\left(\underline{b}_{1} q^{-1}+\underline{b}_{2} q^{-2}\right)^{\mathrm{R}}$. Well, at last? Not quite because the last three inverses include subinverses of (matrix) binomials, each of which can be presented in terms of two 'elementary' inverses involving monomials. The resulting inverses $\underline{B}^{\mathrm{R} 8}\left(q^{-1}\right)$ through $\underline{B}^{\mathrm{R} 13}\left(q^{-1}\right)$ are relegated to Appendix A. Thus, for the above example, as many as 13 different types of right inverses can be involved in 13 various sets of solvers (4) of Eqn. (7) for $u(t)$. And yet, each of those inverses $\underline{B}^{\mathrm{R} 1}\left(q^{-1}\right)$ through $\underline{B}^{\mathrm{R} 13}\left(q^{-1}\right)$ is nonunique due to the nonuniqueness of a right inverse.

In order to arrive at feasible analytical solutions, all the right inverses occurring in $\underline{B}^{\mathrm{R} 1}\left(q^{-1}\right)$ through $\underline{B}^{\mathrm{R} 13}\left(q^{-1}\right)$ are now specialized to what will be called the minimum-norm $T$-inverses and denoted as $[\cdot]_{0}^{\mathrm{R}}$. Since the $\mathrm{MV} /$ perfect control law is clearly a time-domain equation, we shall use regular, rather than conjugate, transposes in the minimum norm $T$-inverse (Latawiec, 2004; Latawiec et al., 2005).

It is interesting to observe that all those inverses are associated with two classes of solvers for $u(t)$ in the MVC problem, resulting from two different equations to be solved: $\underline{B}\left(q^{-1}\right) u(t)=\underline{y}(t)$ and $\left\{I_{n_{u}}+\right.$
$\left.\left[\underline{\beta}_{s}\left(q^{-1}\right)\right]_{0}^{\mathrm{R}}\left[\underline{B}\left(q^{-1}\right)-\underline{\beta}\left(q^{-1}\right)\right]\right\} u(t)=\left[\underline{\beta}_{s}\left(q^{-1}\right)\right]_{0}^{\mathrm{R}} \underline{y}(t)$, where $\underline{\beta}\left(q^{-1}\right)$ and $\underline{\beta}_{s}\left(\bar{q}^{-1}\right)$ can be easily identified from the specific inverses $\underline{B}^{\mathrm{R} 1}\left(q^{-1}\right)$ to $\underline{B}^{\mathrm{R} 13}\left(q^{-1}\right)$. It is worth mentioning that $\underline{\beta}_{s}\left(q^{-1}\right)$ can generally be very complicated (see, e.g., Appendix A), so its general analytical definition would be hardly achievable. Notwithstanding, we will provide means for the computation of the resulting general right inverses $\underline{B}^{\mathrm{R}}\left(q^{-1}\right)=\left\{I_{n_{u}}+\left[\underline{\beta}_{s}\left(q^{-1}\right)\right]_{0}^{\mathrm{R}}\left[\underline{B}\left(q^{-1}\right)-\right.\right.$ $\left.\left.\underline{\beta}\left(q^{-1}\right)\right]\right\}^{-1}\left[\underline{\beta}_{s}\left(q^{-1}\right)\right]_{0}^{\mathrm{R}}$.

\section{MVC-related polynomial matrix inverses}

Let us switch now to more general problems of either right- or left-invertible systems, as well as to a quite general case of non-full normal rank systems with any $\underline{B}\left(q^{-1}\right)$. In general, we will refer to Class 1 and Class 2 solvers for $u(t)$ in the MVC problem, related respectively to the equations

$$
\underline{B}\left(q^{-1}\right) u(t)=\underline{y}(t)
$$

or

$$
\begin{array}{r}
\left\{I_{n_{u}}+\left[\underline{\beta}_{s}\left(q^{-1}\right)\right]^{\mathrm{inv}}\left[\underline{B}\left(q^{-1}\right)-\underline{\beta}\left(q^{-1}\right)\right]\right\} u(t) \\
=\left[\underline{\beta}_{s}\left(q^{-1}\right)\right]^{\mathrm{inv}} \underline{y}(t),
\end{array}
$$

where the inverse $\left[\underline{\beta}_{s}\left(q^{-1}\right)\right]^{\text {inv }}$ is an appropriate generalized inverse of a specific $\underline{\beta}_{s}\left(q^{-1}\right)$, depending on specific, rank-related properties of $\underline{\beta}_{s}\left(q^{-1}\right)$ (with, e.g., $\left[\underline{\beta}_{s}\left(q^{-1}\right)\right]^{\text {inv }}=\left[\underline{\beta}_{s}\left(q^{-1}\right)\right]^{\mathrm{R}}$ holding for a right-invertible $\left.\underline{\beta}_{s}\left(q^{-1}\right)\right)$. Note that for $\underline{\beta}_{s}\left(q^{-1}\right)=\underline{\beta}\left(q^{-1}\right)=\underline{B}\left(q^{-1}\right)$ $\overline{\mathrm{E}} \mathrm{s}$. (10) and (11) are equivalent.

5.1. $T$-inverses. Based on the above considerations, the two general definitions below introduce various optimal inverses of the $m$-th order nonsquare polynomial matrix $\underline{B}\left(q^{-1}\right)$, which are associated with Class 1 optimal time-domain solvers for $u(t)$ in the MVC problem (related to Eqn. (10)). The optimal, so-called $T$-inverses include regular (rather than conjugate) transposes of $\underline{B}\left(q^{-1}\right)$. Observe that these inverses are dimension preserving, i.e., not squaring the system down (Davison, 1983; Latawiec, 1998), the prerequisite aiming at protection from the reduction of the problem to the classical square MIMO one (with the standard transmission zeros).

Definition 1. Let the polynomial matrix $\underline{B}\left(q^{-1}\right)=$ $\underline{b}_{0}+\underline{b}_{1} q^{-1}+\cdots+\underline{b}_{m} q^{-m}$ be of full normal rank $n_{y}$ (or $n_{u}$ ). The (unique) minimum-norm right (or the least-squares left) $T$-inverse of $\underline{B}\left(q^{-1}\right)$ is defined as $\underline{B}_{0}^{\mathrm{R}}\left(q^{-1}\right)=\underline{B}^{\mathrm{T}}\left(q^{-1}\right)\left[\underline{B}\left(q^{-1}\right) \underline{B}^{\mathrm{T}}\left(q^{-1}\right)\right]^{-1}$ (or $\left.\underline{B}_{0}^{\mathrm{L}}\left(q^{-1}\right)=\left[\underline{B}^{\mathrm{T}}\left(q^{-1}\right) \underline{B}\left(q^{-1}\right)\right]^{-1} \underline{B}^{\mathrm{T}}\left(q^{-1}\right)\right)$. 
Definition 2. Let the polynomial matrix $\underline{B}\left(q^{-1}\right)=$ $\underline{b}_{0}+\underline{b}_{1} q^{-1}+\cdots+\underline{b}_{m} q^{-m}$ of non-full normal rank $r$ be skeleton factorized as $\underline{B}\left(q^{-1}\right)=$ $\underline{C}\left(q^{-1}\right) \underline{D}\left(q^{-1}\right)$, where $\operatorname{dim}\left[\underline{B}\left(q^{-1}\right)\right]=n_{y} \times n_{u}$, $\operatorname{dim}\left[\underline{C}\left(q^{-1}\right)\right]=n_{y} \times r, \operatorname{dim}\left[\underline{D}\left(q^{-1}\right)\right]=r \times n_{u}$. The (unique) Moore-Penrose $T$-inverse of $\underline{B}\left(q^{-1}\right)$ is defined as $\underline{B}_{0}^{\#}\left(q^{-1}\right)=\underline{D}_{0}^{\mathrm{R}}\left(q^{-1}\right) \underline{C}_{0}^{\mathrm{L}}\left(q^{-1}\right)$, where $\underline{D}_{0}^{\mathrm{R}}\left(q^{-1}\right)=\underline{D}^{\mathrm{T}}\left(q^{-1}\right)\left[\underline{D}\left(q^{-1}\right) \underline{D}^{\mathrm{T}}\left(q^{-1}\right)\right]^{-1}$ and $\underline{C}_{0}^{\mathrm{L}}\left(q^{-1}\right)=\left[\underline{C}^{\mathrm{T}}\left(q^{-1}\right) \underline{C}\left(q^{-1}\right)\right]^{-1} \underline{C}^{\mathrm{T}}\left(q^{-1}\right)$.

Remark 4. Of course, the above definitions could as well be formulated in the complex $z$-domain, with regular, rather than conjugate, transposes retained and, e.g., $B(z)=z^{m} \underline{B}\left(z^{-1}\right)$. This will also hold for all other polynomial matrix inverses to follow. We still retain the $q^{-1}$ argument to emphasize the time-domain origin of the MVCrelated inverses.

Remark 5. Observe that $T$-inverses have been originally used in the introduction of control zeros, being an extension of transmission zeros (Latawiec, 1998; Latawiec et al., 2000). For example, control zeros for right-invertible systems can be generated by the inverse $\underline{B}_{0}^{\mathrm{R}}\left(q^{-1}\right)$ and calculated from the equation $\operatorname{det}\left[\underline{B}\left(q^{-1}\right) \underline{B}^{\mathrm{T}}\left(q^{-1}\right)\right]=0$. In the sequel, these control zeros will be called control zeros type 1 .

Remark 6. It should be emphasized that the essence of the introduction of such definitions of $T$-inverses is that regular (rather than conjugate) transposes are involved due to the time-domain, MVC-related origin of the inverse problem. When employing conjugate transposes, we end up with transmission zeros only (Latawiec, 2004).

5.2. $\tau$-inverses. Again, we consider a problem of $\underline{B}\left(q^{-1}\right)$ and $\underline{\beta}_{s}\left(q^{-1}\right)$ being either right- or left-invertible, in addition to the general case of $\underline{B}\left(q^{-1}\right)$ having non-full normal rank. Hereinafter, we present detailed results for the right-invertibility case. The right polynomial matrix inverses

$$
\begin{aligned}
& \underline{B}^{\mathrm{R}}\left(q^{-1}\right) \\
& \left.=\left\{I_{n_{u}}+\left[\underline{\beta}_{s}\left(q^{-1}\right)\right]_{0}^{\mathrm{R}} \underline{B}\left(q^{-1}\right)-\underline{\beta}\left(q^{-1}\right)\right]\right\}^{-1}\left[\underline{\beta}_{s}\left(q^{-1}\right)\right]_{0}^{\mathrm{R}},
\end{aligned}
$$

associated with Class 2 time-domain solvers $u(t)$ to Eqn. (11), are now called $\tau$-inverses.

Remark 7. Note that for $\underline{\beta}\left(q^{-1}\right)=\underline{\beta}_{s}\left(q^{-1}\right)=\underline{B}\left(q^{-1}\right)$ the $\tau$-inverse specializes to the $T$-inverse. Still, we distinguish the $T$-inverse from the $\tau$-inverse, at least for 'traditional' reasons.

As mentioned above, we refrain from trying to formally define $\tau$-inverses in terms of the matrices $\underline{\beta}\left(q^{-1}\right)$ and $\underline{\beta}_{s}\left(q^{-1}\right)$ due to very complicated forms of the latter, in general. Nevertheless, based on the motivating example of Section 4, we can offer new general tools for the computation of all the $\tau$-inverses.

\subsubsection{Algorithm and program for calculating} $\tau$-inverses. Below is a new algorithm for calculating all the $\tau$-inverses. The combinatorics-based algorithm is very complicated but, surprisingly, it will self-verifyingly lead to a simple result of the forthcoming Theorem 1 .

Algorithm 1. tau_inverses algorithm.

$$
\text { STEP } k=0 \rightarrow \text { one } T \text {-inverse }
$$$$
\underline{B}_{0}^{\mathrm{R}}\left(q^{-1}\right)=\left\{I_{n_{u}}+\left[\underline{\beta}_{l_{0}}\right]_{0}^{\mathrm{R}}\left[\underline{B}\left(q^{-1}\right)-\underline{\beta}_{l_{0}}\right]\right\}^{-1}\left[\underline{\beta}_{l_{0}}\right]_{0}^{\mathrm{R}},
$$

with $l_{0}=m+1$, that is, $\underline{\beta}_{l_{0}}\left(q^{-1}\right)=\underline{B}\left(q^{-1}\right)$. The index $l_{0}=m+1$ means that matrix parameters of the $m$-th order matrix polynomial $\underline{\beta}_{m+1}\left(q^{-1}\right)$ constitute $(m+1)$-combinations without repetition from an $(m+1)$ parameter set of the matrix polynomial $\underline{B}\left(q^{-1}\right)$, which makes the number of the combinations and thus the number of $T$-inverses equal to just one.

$$
\text { STEP } k=1
$$

$$
\begin{array}{r}
{\left[\underline{B}\left(q^{-1}\right)\right]_{i}^{\mathrm{R}}=\left\{I_{n_{u}}+\left[\underline{\beta}_{l_{1}}^{i}\right]_{0}^{\mathrm{R}}\left[\underline{\beta}_{l_{0}}^{i}-\underline{\beta}_{l_{1}}^{i}\right]\right\}^{-1}\left[\underline{\beta}_{l_{1}}^{i}\right]_{0}^{\mathrm{R}},} \\
i=k, \ldots, m, \\
\forall l_{0}, l_{1} \text { with } l_{0}=1, \ldots, m+1, \\
l_{1}=1, \ldots, m-k+1=m \wedge l_{0} \geq l_{1},
\end{array}
$$

where matrix parameters of the polynomial matrix $\underline{\beta}_{l_{1}}^{i}\left(q^{-1}\right)$ are $l_{1}$-combinations without repetition from an $l_{0^{-}}$parameter set of the matrix polynomial $\underline{\beta}_{l_{0}}^{i}\left(q^{-1}\right)$, and the superscript $i=k, \ldots, m$ stands for the $i$-th set of inverses calculated at Step $k$. The role of the index $i$ will be better understood from the proof of Theorem 1 .

$$
\text { STEP } k=2
$$

$\left[\underline{B}\left(q^{-1}\right)\right]_{i}^{\mathrm{R}}$ as in (A.1) with

$\left[\underline{\beta}_{l_{1}}^{i-1}\right]_{0}^{\mathrm{R}}=\left\{I_{n_{u}}+\left[\underline{\beta}_{l_{2}}^{i}\right]_{0}^{\mathrm{R}}\left[\underline{\beta}_{l_{1}}^{i}-\underline{\beta}_{l_{2}}^{i}\right]\right\}^{-1}\left[\underline{\beta}_{l_{2}}^{i}\right]_{0}^{\mathrm{R}}$,

$$
i=k, \ldots, m,
$$

$$
\forall l_{1}, l_{2} \text { with } l_{1}, l_{2}=1, \ldots, m-k+1=m-1 \wedge
$$

with the notation quite similar to that for Step $k=1$.

\section{STEP $k$}

$\left[\underline{B}\left(q^{-1}\right)\right]_{i}^{\mathrm{R}}$ as in $(\mathrm{A} . k-1)$ with $\left[\underline{\beta}_{l_{k-1}}^{i-1}\right]_{0}^{\mathrm{R}}=\left\{I_{n_{u}}+\left[\underline{\beta}_{l_{k}}^{i}\right]_{0}^{\mathrm{R}}\left[\underline{\beta}_{l_{k-1}}^{i}-\underline{\beta}_{l_{k}}^{i}\right]\right\}^{-1}\left[\underline{\beta}_{l_{k}}^{i}\right]_{0}^{\mathrm{R}}$, 


$$
\begin{array}{r}
i=k, \ldots, m, \\
\forall l_{k-1}, l_{k} \text { with } l_{k-1}, l_{k}=1, \ldots, m-k+1 \wedge \\
l_{k-1} \geq l_{k} .
\end{array}
$$

$$
\text { STEP } k=m-1
$$

$\left[\underline{B}\left(q^{-1}\right)\right]_{i}^{\mathrm{R}}$ as in $($ A. $m-2)$ with

$\left[\underline{\beta}_{m-2}^{i-1}\right]_{0}^{\mathrm{R}}$

$$
=\left\{I_{n_{u}}+\left[\underline{\beta}_{l_{m-1}}^{i}\right]_{0}^{\mathrm{R}}\left[\underline{\beta}_{l_{m-2}}^{i}-\underline{\beta}_{l_{m-1}}^{i}\right]\right\}^{-1}\left[\underline{\beta}_{l_{m-1}}^{i}\right]_{0}^{\mathrm{R}},
$$

$$
i=m-1, m \text {, }
$$

$\forall l_{m-2}, l_{m-1}$ with $l_{m-2}, l_{m-1}=1,2$

$$
\wedge l_{m-2} \geq l_{m-1} \text {. }
$$

$($ A. $m-1)$

STEP $k=m$

$\left[\underline{B}\left(q^{-1}\right)\right]_{i}^{\mathrm{R}}$ as in $($ A. $m-1)$ with

$$
\begin{array}{r}
{\left[\underline{\beta}_{l_{m-1}}^{i-1}\right]_{0}^{\mathrm{R}}=\left\{I_{n_{u}}+\left[\underline{\beta}_{l_{m}}^{i}\right]_{0}^{\mathrm{R}}\left[\underline{\beta}_{l_{m-1}}^{i}-\underline{\beta}_{l_{m}}^{i}\right]\right\}^{-1}\left[\underline{\beta}_{l_{m}}^{i}\right]_{0}^{\mathrm{R}},} \\
i=m, \\
\forall l_{m-1}, l_{m} \text { with } l_{m-1}=l_{m}=1 .
\end{array}
$$

(A.m)

The algorithm is coded using the Symbolic Toolbox ${ }^{\circledR}$, Polynomial Toolbox ${ }^{\circledR}$ and Statistics Toolbox ${ }^{\circledR}$ in the Matlab ${ }^{\circledR}$ environment. The program returns all the $\tau$-inverses and the associated sets of control zeros. The codes for this program as well as for all other programs exploited in the paper can be made available upon request.

Theorem 1. (Latawiec, 2004) Consider a nonsquare full normal rank polynomial matrix $\underline{B}\left(q^{-1}\right)=\underline{b}_{0}+\underline{b}_{1} q^{-1}+$ $\ldots+\underline{b}_{m} q^{-m}$. The total number $N_{m}$ of the $\tau$-inverses of $\underline{B}\left(q^{-1}\right)$ can be calculated iteratively from the equation

$$
\begin{array}{r}
N_{i}=1+(i+1) ! \sum_{j=1}^{i} \frac{1}{j !(i-j+1) !} N_{j-1}, \\
i=1, \ldots, m, \quad N_{0}=1 .
\end{array}
$$

Proof. See Appendix B.

Remark 8. Although Theorem 1 has been presented by Latawiec (2004), it is not until this paper that an original, complete, formal proof has been provided.

Remark 9. The above total number of $\tau$-inverses shall be treated as the maximum number of $\tau$-inverses for a specific $m$. In fact, in some cases $\underline{\beta}\left(q^{-1}\right)$ and/or $\underline{\beta}_{s}\left(q^{-1}\right)$ may appear nonfull normal rank (even though $\underline{B}\left(q^{-1}\right)$ is of full normal rank) so that the corresponding $\tau$-inverses do not exist. Exemplary maximum numbers of $\tau$-inverses are $N_{m}=13$ for $m=2, N_{m}=75$ for $m=3$ and $N_{m}=541$ for $m=4$.

Remark 10. It has been found in simulations (a rigorous mathematical confirmation seems unlikely) that for some, even 'typical', plants the properties of $\tau$-inverses of $\underline{B}\left(q^{-1}\right)$, including the $T$-inverse, may be unfavorable, in particular in terms of all unstable poles of $\underline{B}^{\mathrm{R}}\left(q^{-1}\right)$ obtained. This may limit the applications of $\tau$-inverses.

5.3. $\sigma$-inverses. Let us proceed now to the most intriguing issue related to the family of inverses as in Eqn. (12). It is surprising that $\underline{B}\left(q^{-1}\right) \underline{B}^{\mathrm{R}}\left(q^{-1}\right)=I_{n_{y}}$, with $\underline{B}^{\mathrm{R}}$ as in Eqn. (12) and $\underline{\beta}_{s}\left(q^{-1}\right)=\underline{\beta}\left(q^{-1}\right)$, even for arbitrary $\beta\left(q^{-1}\right)$, that is, not related to $\underline{B}\left(q^{-1}\right)$ at all (but, of course, with adequate matrix dimensions). This way we arrive at the so-called $\sigma$-inverses, a number of which is infinite (in spite of the unique minimum-norm right $T$-inverse involved).

Definition 3. Let the polynomial matrix $\underline{B}\left(q^{-1}\right)=\underline{b}_{0}+$ $\underline{b}_{1} q^{-1}+\cdots+\underline{b}_{m} q^{-m}$. Then, a general $\sigma$-inverse of $\underline{B}\left(\underline{q}^{-1}\right)$ can be defined as

$$
\begin{aligned}
& \underline{B}^{\mathrm{R}}\left(q^{-1}\right) \\
& =\left\{I_{n_{u}}+\left[\underline{\beta}\left(q^{-1}\right)\right]_{0}^{\mathrm{R}}\left[\underline{B}\left(q^{-1}\right)-\underline{\beta}\left(q^{-1}\right)\right]\right\}^{-1}\left[\underline{\beta}\left(q^{-1}\right)\right]_{0}^{\mathrm{R}},
\end{aligned}
$$

where $z^{s} \beta\left(z^{-1}\right)=\beta(z) \in \mathbb{R}^{n_{y} \times n_{u}}[z]$ is arbitrary, including an arbitrary order $s$, and $[\cdot]_{0}^{\mathrm{R}}$ stands for the $T$-inverse.

Unfortunately, rigorous formal proving that $\underline{B}\left(q^{-1}\right) \underline{B}^{\mathrm{R}}\left(q^{-1}\right)=I_{n_{y}}$, with $\underline{B}^{\mathrm{R}}\left(q^{-1}\right)$ as in Eqn. (14), is still an open problem. A missing part of the proof can be formulated as follows.

Conjecture 1. Let $\beta\left(q^{-1}\right)$ be an arbitrary s-order matrix polynomial in $q^{-1}$, with $z^{s} \underline{\beta}\left(z^{-1}\right)=\beta(z) \in \mathbb{R}^{n_{y} \times n_{u}}[z]$, and let $\Phi\left(q^{-1}\right)=I_{n_{u}}+\left[\underline{\beta}\left(q^{-1}\right)\right]_{0}^{\mathrm{R}}\left[\underline{B}\left(q^{-1}\right)-\underline{\beta}\left(q^{-1}\right)\right]$ be of full normal rank $n_{u}$. Then

$$
\left[\underline{\beta}\left(q^{-1}\right) \Phi\left(q^{-1}\right)\right]_{0}^{\mathrm{R}}=\Phi^{-1}\left(q^{-1}\right)\left[\underline{\beta}\left(q^{-1}\right)\right]_{0}^{\mathrm{R}} .
$$

Assuming that the above conjecture is true, the proof of $\underline{B}\left(q^{-1}\right) \underline{B}^{\mathrm{R}}\left(q^{-1}\right)=I_{n_{y}}$, with $\underline{B}^{\mathrm{R}}\left(q^{-1}\right)$ as in Eqn. (14), is immediate. In fact, $\beta\left(q^{-1}\right) \Phi\left(q^{-1}\right)=\underline{B}\left(q^{-1}\right)$ whereas the right-hand side of Eqn. (15) is just the right-hand side of Eqn. (14). Now, omitting the subindex on the left-hand side of Eqn. (15) (in order to distinguish the $\sigma$-inverse from the $T$-inverse) would complete the proof.

Remark 11. We have verified Conjecture 1 and the identity $\underline{B}\left(q^{-1}\right) \underline{B}^{\mathrm{R}}\left(q^{-1}\right)=I_{n_{y}}$, with $\underline{B}^{\mathrm{R}}\left(q^{-1}\right)$ as in Eqn. (14), in a number of simulations, including MVC ones. 
Remark 12. It is interesting to note here that, for arbitrary right-invertible $\beta\left(q^{-1}\right)$ and arbitrary invertible $\Phi\left(q^{-1}\right)$ of appropriate dimensions, we have that $\left[\Phi\left(q^{-1}\right) \beta\left(q^{-1}\right)\right]_{0}^{\mathrm{R}}=\left[\beta\left(q^{-1}\right)\right]_{0}^{\mathrm{R}} \Phi^{-1}\left(q^{-1}\right)$, but $\left[\beta\left(q^{-1}\right) \Phi\left(q^{-1}\right)\right]_{0}^{\overline{\mathrm{R}}} \neq \Phi^{-1}\left(q^{-1}\right)\left[\beta\left(q^{-1}\right)\right]_{0}^{\mathrm{R}}$, in general. It is just for our specific $\bar{\Phi}\left(q^{-1}\right)=I_{n_{u}}+$ $\left[\underline{\beta}\left(q^{-1}\right)\right]_{0}^{\mathrm{R}}\left[\underline{B}\left(q^{-1}\right)-\underline{\beta}\left(q^{-1}\right)\right]$ that Eqn. (15) holds true, the intriguing property confirmed so far only in numerous simulations.

Even though the most general $\sigma$-inverses contain $\tau$ inverses, which in turn include the $T$-inverse, we discriminate between the three types of inverses of nonsquare polynomial matrices. Here $\tau$-inverses and $\sigma$-inverses generate what we call control zeros type 2 (Latawiec, 2004; Latawiec et al., 2004).

It is worth emphasizing that the formula (14) is a new, general, algorithmically very simple analytical expression for the calculation of right inverses for nonsquare polynomial matrices. Quite a similar formula can be given for left inverses. It is interesting to note how stimulating the MVC framework in the derivation of $\tau$ - and $\sigma$-inverses has been (Latawiec, 2004; Latawiec et al., 2004; 2005).

Remark 13. Of course, the inverse as in Eqn. (14) can be rewritten in terms of $B(z)=z^{m} \underline{B}\left(z^{-1}\right)$. We still prefer the form (14) associated with the time-domain MVC solution and the regular, rather than conjugate, transposes included in $\left[\beta\left(q^{-1}\right)\right]_{0}^{\mathrm{R}}$. Notwithstanding, the $z$-domain formulation is now used instructively below.

Example 1. Consider a specific matrix $\underline{B}\left(q^{-1}\right)$ corresponding to the matrix $B \in \mathbb{R}^{2 \times 3}[z]$ with $b_{11}(z)=$ $z^{2}+0.9 z-0.1, b_{12}(z)=z+0.4, b_{13}(z)=z^{2}+$ $0.1 z-0.02$ and $b_{21}(z)=z^{2}+0.4 z-0.05, b_{22}(z)=1$, $b_{23}(z)=2 z^{2}+0.8 z-0.1$. All the thirteen $\tau$-inverses (including the $T$-inverse) have unstable poles, that is, unstable control zeros, in addition to a stable transmission zero at $z=0.1$. Selecting $\beta_{s}=\beta \in \mathbb{R}^{2 \times 3}[z]$ with $\beta_{11}(z)=-0.0793, \beta_{12}(z)=1.5352 z-0.6065$, $\beta_{13}(z)=-1.3474$ and $\beta_{21}(z)=0.4694 z-0.9036$, $\beta_{22}(z)=0.0359, \beta_{23}(z)=-0.6275 z+0.5354$ yields the unstable $\sigma$-inverse $B^{\mathrm{R}}(z)$, whereas for $\beta_{11}(z)=$ $-0.0781, \beta_{12}(z)=1.8148 z-0.6140, \beta_{13}(z)=-1.3928$ and $\beta_{21}(z)=0.3931 z-0.6786, \beta_{22}(z)=0.0332$, $\beta_{23}(z)=-0.8042 z+0.6203$ we obtain the stable $\sigma$ inverse $B^{\mathrm{R}}(z)$. However, there are no formal tools for rigorous generalization of the latter, heuristic selection.

For a specific $\underline{B}\left(q^{-1}\right)$, we have computed an adequate $\beta\left(q^{-1}\right)$ to obtain a stable (or pole-free) $\underline{B}^{\mathrm{R}}\left(q^{-1}\right)$ by means of a standard, Matlab-based optimization procedure. (Note that, for space limitation reasons, we refrain from specifying the two $\sigma$-inverses $B^{\mathrm{R}}(z)$ in the above example as their six entries are up to the 5 -th order, so that high-precision presentation would be necessary for accuracy reasons. Still, in Appendix $C$ we specify control zeros, that is, poles of $B^{\mathrm{R}}(z)$, for the two cases.)

\section{New approaches to stable design of inverse polynomial matrices}

It is crucial in inverse-model control applications to be able to design stable inverses of the numerator matrix in the rational matrix description of an LTI MIMO system. Particular interest concerns the case of pole-free inverses, for which the control system is guaranteed to be asymptotically stable. Here we present two new approaches to the design of pole-free right inverses of a polynomial matrix $\underline{B}\left(q^{-1}\right)$.

6.1. Extreme Points and Extreme Directions (EPED) method. The method is recalled here for solving the linear matrix polynomial equation (Callier and Kraffer, 2005; Henrion, 1998; Kaczorek and Łopatka, 2000)

$$
K(w) X(w)=P(w),
$$

where $K(w)=K_{0}+K_{1} w+\cdots+K_{n_{K}} w^{n_{K}}$ and $P(w)=$ $P_{0}+P_{1} w+\cdots+P_{n_{P}} w^{n_{P}}$ are given $m \times n$ and $m \times p$ polynomial matrices in complex operator $w$, respectively, and $X(w)=X_{0}+X_{1} w+\cdots+X_{n_{X}} w^{n_{X}}$ is an $n \times p$ polynomial matrix to be found. By equating the powers of $w$ in the formula (16), we obtain an equivalent linear system of equations

$$
\bar{K} \bar{X}=\bar{P}
$$

where the real matrix

$$
\bar{K}=\left[\begin{array}{cccc}
K_{0} & & & 0 \\
K_{1} & K_{0} & & \\
\vdots & K_{1} & \ddots & \\
K_{n_{K}} & \vdots & & K_{0} \\
& K_{n_{K}} & & K_{1} \\
& & \ddots & \vdots \\
0 & & & K_{n_{K}}
\end{array}\right]
$$

is referred to as the Sylvester matrix of $K(w)$ of order $n_{K}$, with $\tilde{m}=\left(n_{K}+n_{X}+1\right) m$ rows and $\tilde{n}=\left(n_{X}+1\right) n$ columns and

$$
\bar{X}=\left[\begin{array}{c}
X_{0} \\
X_{1} \\
\vdots \\
X_{n_{X}}
\end{array}\right] \in \mathbb{R}^{\tilde{n} \times p}, \quad \bar{P}=\left[\begin{array}{c}
P_{0} \\
P_{1} \\
\vdots \\
P_{n_{P}}
\end{array}\right] \in \mathbb{R}^{\tilde{m} \times p} .
$$

The problem of finding the matrix polynomial solution $X(w)$ to Eqn. (16) has been reduced to finding the real matrix $\bar{X}$ of Eqn. (17) for given real matrices $\bar{K}$ and $\bar{P}$ as in (18) and (19). The matrix polynomial equation 16 has the solution for $X(w)$ iff $\operatorname{rank}\left[\begin{array}{cc}\bar{K} & \bar{P}\end{array}\right]=\operatorname{rank} \bar{K}$. Using the Kronecker product, Eqn. (17) can be rewritten in the form

$$
A x=b,
$$


where

$$
\begin{gathered}
A=\bar{K} \otimes I_{p} \in \mathbb{R}^{\bar{m} \times \bar{n}}, \\
x=\left[\begin{array}{cccc}
\bar{x}_{1}, & \bar{x}_{2}, & \ldots, & \bar{x}_{\tilde{n}}
\end{array}\right]^{\mathrm{T}} \in \mathbb{R}^{\bar{n}}, \\
b=\left[\begin{array}{cccc}
\bar{p}_{1}, & \bar{p}_{2}, & \ldots, & \bar{p}_{\tilde{m}}
\end{array}\right]^{\mathrm{T}} \in \mathbb{R}^{\bar{m}},
\end{gathered}
$$

with $\bar{m}=\tilde{m} p, \bar{n}=\tilde{n} p$, and $\bar{x}_{i}$ and $\bar{p}_{j}$ denote the $i$-th and $j$-th rows of $\bar{X}$ and $\bar{P}$, respectively.

Now, the problem of calculating the set of solutions to Eqn. (16) can be reduced to finding the set $x$ satisfying Eqn. (20). Note that, if $\tilde{n} \geq \tilde{m}$ and $\operatorname{rank} \bar{K}=\tilde{m}$, the matrix $A$ also has full row rank.

Let $S=\{x: A x=b\}$ be a non-empty set. A point $x$ is an extreme point of $S$ iff $A$ can be decomposed into $\left[\begin{array}{ll}B & N\end{array}\right]$ such that $\operatorname{det} B \neq 0$ and $x=\left[\begin{array}{c}B^{-1} b \\ 0\end{array}\right]$. If $\operatorname{rank} A=\bar{m}$, then $S$ has at least one extreme point. The number of extreme points is less than or equal to $\bar{n} ! /[\bar{m} !(\bar{n}-\bar{m}) !]$.

A vector $d$ is an extreme direction of $S$ iff $A$ can be decomposed into $[B N]$ such that $\operatorname{det} B \neq 0$ and $d=$ $\left[\begin{array}{c}-B^{-1} a_{j} \\ e_{j}\end{array}\right]$, where $a_{j}$ is the $i$-th column of $N$ and $e_{j}$ is an $\bar{n}-\bar{m}$ vector of zeros except for unity in position $i$. The set $S$ has at least one extreme direction iff it is unbounded. The maximum number of extreme directions is bounded by $\bar{n} ! /[\bar{m} !(\bar{n}-\bar{m}-1) !]$.

Let $x_{1}, x_{2}, \ldots, x_{k}$ be the extreme points of $S$ and $d_{1}$, and $d_{2}, \ldots, d_{l}$ the extreme directions of $S$. Then every $x \in S$ can be written as

$$
x=\sum_{j=1}^{k} \lambda_{j} x_{j}+\sum_{i=1}^{l} \mu_{i} d_{i}, \quad \sum_{j=1}^{k} \lambda_{j}=1 .
$$

Let us now embed the EPED method in the framework of an inverse polynomial matrix $\underline{B}^{\mathrm{R}}\left(z^{-1}\right)$.

Theorem 2. Let $\underline{B}^{\mathrm{R}}(w)=X(w)$, with $w=z^{-1}$, be a solution of the linear matrix polynomial equation $\underline{B}(w) X(w)=I_{n_{y}}$. Then the necessary and sufficient condition for the existence of a solution $X(w)$ by the EPED method is that the polynomial matrix $\underline{B}(w)$ has no transmission zeros.

Proof. It is well known that the necessary and sufficient condition for the existence of a solution of Eqn. (16) is that $\operatorname{nrank}\left[\begin{array}{ll}K(w) & P(w)\end{array}\right]=\operatorname{nrank} K(w)$, where nrank stands for normal rank. When translated to our polynomial matrix framework, the condition $\operatorname{nrank}\left[\underline{B}(w) \quad I_{n_{y}}\right]=\operatorname{nrank} \underline{B}(w)$ implies that the matrix $\underline{B}(w)$ has no transmission zeros.

The above essential constraint of the EPED method is revealed here for the first time.

The mathematically elegant EPED method provides a pole-free solution to the inverse polynomial matrix problem, but it is computationally involving while its use is limited to systems with no transmission zeros. Therefore, in the next section we offer a much simpler method, which is valid for systems with transmission zeros as well. Even if the method is more effective, it is the EPED method which has turned our attention to pole-free design of inverse polynomial matrices.

6.2. Smith factorization approach. In an attempt to essentially reduce the computational burden of the EPED method, we introduce yet another effective (and much simpler) approach to stable design of inverse polynomial matrices. The applied approach is closely related to the Smith-McMillan theory (Bose and Mitra, 1978; Kailath, 1980; Sontag, 1980; Vardulakis, 1991).

Consider a right-invertible polynomial matrix $\underline{B}\left(z^{-1}\right)$ of dimension $n_{y} \times n_{u}$. Set $w=z^{-1}$ and factorize $\underline{B}(w)$ to the Smith form $\underline{B}(w)=U(w) S(w) V(w)$, where $U(w)$ and $V(w)$ are (nonunique) unimodular matrices. Now, $\underline{B}^{\mathrm{R}}(w)=V^{-1}(w) S^{\mathrm{R}}(w) U^{-1}(w)$, with determinants of $U(w)$ and $V(w)$ being independent of $w$, that is, with possible instability of an inverse polynomial matrix $\underline{B}^{\mathrm{R}}(w)$ being related to $S^{\mathrm{R}}(w)$ only.

Theorem 3. Consider a right-invertible polynomial $n_{y} \times n_{u}$ matrix $\underline{B}\left(z^{-1}\right)$. Use the Smith factorization and obtain the inverse polynomial matrix $\underline{B}^{\mathrm{R}}(w)=$ $V^{-1}(w) S^{\mathrm{R}}(w) U^{-1}(w)$, with $w=z^{-1}$ and $U(w)$ and $V(w)$ being unimodular. Then, applying the minimumnorm right T-inverse $S_{0}^{\mathrm{R}}(w)=S^{\mathrm{T}}(w)\left[S(w) S^{\mathrm{T}}(w)\right]^{-1}$ guarantees stable pole-free design of $\underline{B}^{\mathrm{R}}(w)$ for $\underline{B}(w)$ without transmission zeros and stable design of $\underline{B}^{\overline{\mathrm{R}}}(w)$ for $\underline{B}(w)$ with stable transmission zeros.

Proof. Observe that performing the Smith factorization for $\underline{B}(w)$ one obtains $\underline{B}(w)=U(w) S(w) V(w)$, where $\bar{U}(w)$ and $V(w)$ are unimodular. Now, $\underline{B}^{\mathrm{R}}(w)=$ $V^{-1}(w) S^{\mathrm{R}}(w) U^{-1}(w)$, with determinants of $U(w)$ and $V(w)$ being independent of $w$, that is with possible instability of an inverse polynomial matrix $\underline{B}^{\mathrm{R}}(w)$ being related to $S^{\mathrm{R}}(w)$ only. Since, in general, $S(w)=$ $\left[\operatorname{diag}\left(\varepsilon_{1}, \ldots, \varepsilon_{n_{y}}\right) \quad 0_{n_{y} \times\left(n_{u}-n_{y}\right)}\right]=S_{t z}(w) \underline{S}$, where $S_{t z}(w)=\operatorname{diag}\left(\varepsilon_{1}, \ldots, \varepsilon_{n_{y}}\right)$ includes transmission zeros and $\underline{S}=\left[\begin{array}{ll}I_{n_{y}} & 0_{n_{y} \times\left(n_{u}-n_{y}\right)}\end{array}\right]$, we have $\underline{B}^{\mathrm{R}}(w)=V^{-1}(w) \underline{S}^{\mathrm{R}} S_{t z}^{-1}(w) U^{-1}(w)$. Now $\underline{S}_{0}^{\mathrm{R}}=$ $\underline{S}^{\mathrm{T}}\left[\underline{S} \underline{S}^{\mathrm{T}}\right]^{-1}=\left[\begin{array}{ll}I_{n_{y}} & 0_{n_{y} \times\left(n_{u}-n_{y}\right)}\end{array}\right]^{\mathrm{T}}$ and the result follows immediately.

Remark 14. Obviously, the stability of an inverse polynomial matrix with respect to $w$ is translated to the requirement for all its poles to lie outside the unit disk.

Remark 15. MVC applications of the above Theorems 2 and 3 are immediate. 


\section{Stable Smith factorization design of inverse polynomial matrices with arbitrary degrees of freedom}

In the previous section, stable inverse polynomial matrix designs were obtained without any reference to a possible infinite number of degrees of freedom, which can be of interest in, e.g., control robustness considerations (Hunek, 2008; 2009a; 2009b; Hunek et al., 2007; Latawiec, 2004; 2005; Latawiec et al., 2004; 2005). Even though the unimodular matrices involved are nonunique, possible use of the resulting degrees of freedom is rather difficult due to the constraints imposed on matrix determinants. Here we present a simple Smith factorization approach to pole-free design of inverse polynomial matrices (or pole-free MVC design) with arbitrary degrees of freedom and controlled locations of (stable) zeros of $\underline{B}^{\mathrm{R}}(w)$. Recall that

$$
\underline{B}^{\mathrm{R}}(w)=V^{-1}(w) \underline{S}^{\mathrm{R}} S_{t z}^{-1}(w) U^{-1}(w) .
$$

With a specific form of $\underline{S}=\left[\begin{array}{ll}I_{n_{y}} & 0_{n_{y} \times\left(n_{u}-n_{y}\right)}\end{array}\right]$, we can immediately offer its arbitrary right inverse,

$$
\underline{S}^{\mathrm{R}}=\underline{S}^{\mathrm{R}}(w)=\left[\begin{array}{c}
I_{n_{y}} \\
L(w)
\end{array}\right],
$$

where $L(w)$ is any polynomial matrix of the appropriate dimensions. A general form of that matrix can be $L(w)=\left\{l_{i j}(w)\right\}, i=1, \ldots, n_{u}-n_{y}, j=1, \ldots, n_{y}$, with $l_{i j}(w)=l_{i j}^{(0)}+l_{i j}^{(1)} w+\cdots+l_{i j}^{\left(m_{i j}\right)} w^{m_{i j}}$, and $m_{i j}$ can be an arbitrary natural number selected by the designer.

A general selection of a 'proper' $L(w)$ to provide arbitrary zeros of $\underline{B}^{\mathrm{R}}(w)$ is a difficult, still open problem. In fact, the zeros of $\underline{B}^{\mathrm{R}}(w)$ result both from those of $L(w)$ and those of inverses of the two unimodular polynomial matrices involved. Specifically, the 'zero matrix polynomial' for $\underline{B}^{\mathrm{R}}(w)$ is $Z(w)=\operatorname{adj} V(w) \underline{S}^{\mathrm{R}}(w) \operatorname{adj} U(w)$, with $\underline{S}^{\mathrm{R}}(w)=\left[\begin{array}{c}I_{n_{y}} \\ L(w)\end{array}\right]$, and $L(w)$ is a polynomial matrix as in Eqn. (22). (Note that $Z \in \mathbb{R}^{n_{u} \times n_{y}}[w]$.) The zeros of $Z(w)$ are the poles of a left inverse $Z^{\mathrm{L}}(w)$, with the myriads of possible polynomial matrix inverses involved again. The role of $L(w)$ in controlling the stability of zeros of $\underline{B}^{\mathrm{R}}(w)$ can be appreciated from what follows.

Example 2. Consider a specific matrix $\underline{B} \in \mathbb{R}^{2 \times 3}(w)$ with

$$
\underline{b}_{0}=\left[\begin{array}{ccc}
2 & 3 & 1 \\
0 & 0 & -1
\end{array}\right], \quad \underline{b}_{1}=\left[\begin{array}{ccc}
-2 & -1 & -2 \\
1 & 1 & 0.1
\end{array}\right]
$$

and

$$
\underline{b}_{2}=\left[\begin{array}{ccc}
1 & 2 & 3 \\
-0.5 & -1.9 & -3
\end{array}\right] .
$$

Pursuing the inverse $\underline{B}^{\mathrm{R}}(w)$ according to Eqns. (21) and (22), we compare two cases: (i) $L(w)=\left[\begin{array}{cc}0 & 0\end{array}\right]$ and (ii) $L(w)=\left[\begin{array}{ll}-1.5189 & 0.6388\end{array}\right]$. The matrices $Z(w)$ for the two cases are respectively $Z_{1}(w)$ and $Z_{2}(w)$, both relegated to Appendix D. We can employ various proposed left inverses to obtain $Z^{\mathrm{L}}(w)$. However, we choose the simple $T$-inverse $Z_{0}^{\mathrm{L}}(w)=\left[Z^{\mathrm{T}}(w) Z(w)\right]^{-1} Z^{\mathrm{T}}(w)$ as it will generate poles of $Z^{\mathrm{L}}(w)$, that is, zeros of $\underline{B}^{\mathrm{R}}(w)$ to be controlled by means of $L(w)$. Now, in the first case, we can see that two out of eight zeros of $Z_{1}(w)$ are unstable, whereas for $Z_{2}(w)$ all the ten zeros are strictly stable (with all their modules with respect to $z=$ $w^{-1}$ being lower than unity). It is worth mentioning that the parameters of $L(w)$ in the second case result from a numerical optimization procedure in which the sum of modules of all the zeros (in terms of $z$ ) is minimized with respect to the parameters $a$ and $b$ contained in $L(w)=\left[\begin{array}{ll}a & b\end{array}\right]$.

Remark 16. Note that the solution

$$
\underline{S}^{\mathrm{R}}=\underline{S}^{\mathrm{R}}(w)=\left[\begin{array}{c}
I_{n_{y}} \\
L(w)
\end{array}\right]
$$

can as well be obtained by the EPED method, with $K(w)=\underline{S}, X(w)=\underline{S}^{\mathrm{R}}$ and $P(w)=I_{n_{y}}$, although under a higher computational burden.

Remark 17. It is worth mentioning once more that all the above designs guarantee the stability of $B^{\mathrm{R}}(z)=$ $z^{-m} \underline{B}^{\mathrm{R}}\left(z^{-1}\right)$ both in case of the lack of transmission zeros and under stable transmission zeros, with control zeros (other than transmission zeros) totally eliminated.

Remark 18. It is the right $T$-inverse applied to the matrix $\underline{S}$ that allows eliminating control zeros according to Theorem 3. Applying some other inverses, that is, $\tau$ - and $\sigma$-inverses, usually ends up with control zeros. However, our simulating experience shows that it is sometimes possible to choose a matrix polynomial $\underline{\beta}\left(z^{-1}\right)$ in Eqn. (14) such that pole-free or stable-pole $\sigma$-inverse(s) of $\underline{B}\left(z^{-1}\right)$ can be obtained. A general selection of such $\sigma$-inverses is a very difficult and still open problem. Therefore, it seems that an inverse of the $\underline{B}\left(z^{-1}\right)$ matrix, based on the Smith factorization as in Eqn. (21), can be more useful in stable design of inverse polynomial matrices.

Remark 19. The question arises whether in some control applications the pole-free, that is, control zero-free inverse polynomial matrix designs could be inferior to the synthesis allowing for (stable) poles of the control system, that is, (stable) control zeros selected to provide, e.g., a sort of robustness to MVC. Such a solution is presented in Section 8.

Remark 20. It is in general possible in the above stable Smith factorization design to select $L(w)$ as a (stable) rational matrix or, in a technically simpler way, as a matrix with all its elements being (stable) rational transfer functions, with an infinite number of degrees of freedom possible to be obtained from degree(s) of the transfer functions. 
Then the poles of those transfer functions are the poles of an inverse polynomial matrix $\underline{B}^{\mathrm{R}}(w)$ or the poles of a closed-loop MVC system, that is, the control zeros. Alternatively, the control zeros (together with transmission zeros) can be generated as poles of $\underline{B}^{\mathrm{R}}(w)$ using Eqn. (14), with an infinite number of degrees of freedom obtained from parameter matrices and degree(s) of $\beta(w)$.

Remark 21. For high-order $\underline{B}(w)$ and $L(w)$, the computation burden of the optimization procedure may become prohibitively high.

\section{New inverse of a nonsquare polynomial matrix}

Concluding the Smith factorization approach to the design of (stable) inverse polynomial matrices, we can offer yet another, general right inverse of a nonsquare polynomial matrix $\underline{B}\left(z^{-1}\right)$, which can be competitive to that of Eqn. (14) and which we call an $S$-inverse. The new result, following immediately from Theorem 3 and Section 7 , is given in the form below.

Corollary 1. Consider a polynomial $n_{y} \times n_{u}$ matrix $\underline{B}(w)$ of full normal rank $n_{y}$, with $w=z^{-1}$, under the Smith factorization $\underline{B}(w)=U(w) S(w) V(w)$, where $U(w)$ and $V(w)$ are unimodular and $S(w)=$ $\left[\operatorname{diag}\left(\varepsilon_{1}, \ldots, \varepsilon_{n_{y}}\right) \quad 0_{n_{y} \times\left(n_{u}-n_{y}\right)}\right]$. Then a general right inverse of $\underline{B}(w)$ can be given as

$$
\underline{B}^{\mathrm{R}}(w)=V^{-1}(w) \underline{S}^{\mathrm{R}}(w) S_{t z}^{-1}(w) U^{-1}(w),
$$

where $S_{t z}(w)=\operatorname{diag}\left(\varepsilon_{1}, \ldots, \varepsilon_{n_{y}}\right)$, with $\varepsilon_{i}$ being the invariant factors, and

$$
\underline{S}^{\mathrm{R}}(w)=\left[\begin{array}{c}
I_{n_{y}} \\
L(w)
\end{array}\right],
$$

where $L \in \mathbb{R}^{\left(n_{u}-n_{y}\right) \times n_{y}}(w)$ is an arbitrary rational matrix.

The above Corollary 1 is in fact a definition of new, general $S$-inverses of nonsquare polynomial matrices. The problem arises with the selection of $L(w)$ to provide arbitrary locations of poles and zeros of $\underline{B}^{\mathrm{R}}(w)$, which can be useful in inverse-model control designs (Hunek, 2009a; 2009b) and, possibly, in error-control coding where up-to-date applications include the case $L(w)=0$ only (Fornasini and Pinto, 2004).

Quite simple is a selection providing arbitrary (possibly stable) poles of $\underline{B}^{\mathrm{R}}(w)$. In fact, selecting the MFD

$$
L(w)=M^{-1}(w) N(w),
$$

with $M \in \mathbb{R}^{\left(n_{u}-n_{y}\right) \times\left(n_{u}-n_{y}\right)}$ and $N \in \mathbb{R}^{\left(n_{u}-n_{y}\right) \times n_{y}}$, reduces the problem to choosing such an $M(w)$ for which the roots of $\operatorname{det} M(w)$ are preselected. Even simpler is here the selection

$$
M(w)=m(w) I_{n_{u}-n_{y}},
$$

where a scalar polynomial $m(w)$ can be arbitrary, both in terms of its order and parameter values.

A general solution for $N(w)$ providing arbitrary zeros of $\underline{B}^{\mathrm{R}}(w)$ is an open problem. Still, we can use the same arguments as for the selection of $L(w)$ in Section 7 and state that zeros of $\underline{B}^{\mathrm{R}}(w)$ can be assigned to some controlled, possibly stable locations by means of $N(w)$.

\section{Actual and potential applications}

Actually, our interest in right/left inverses of polynomial matrices has evolved from MV/perfect control applications (Hunek and Latawiec, 2009; Latawiec, 1998; Latawiec et al., 2000) which are now being expanded towards robust MV/perfect control (Hunek, 2008; 2009a; Hunek et al., 2010; 2007; Latawiec, 2004; 2005; Latawiec et al., 2004; 2005). Quite similar families of applications include distortionless (or perfect) reception in error-control coding (Fornasini and Pinto, 2004; Forney, 1991; GluesingLuerssen et al., 2006; Johannesson and Zigangirov, 1999; Johannesson et al., 1998; Lin and Costello, 2004; Lu et al., 2005; Moon, 2005), perfect precoding and equalization (Boche and Pohl, 2006; Guo and Levy, 2004; Kim and Park, 2004; Tidestav et al., 1999; Wahls and Boche, 2007; Wahls et al., 2009; Xia et al., 2001), perfect signal reconstruction, including perfect reconstruction filter banks (Bernardini and Rinaldo, 2006; Gan and Ling, 2008; Law et al., 2009; Quevedo et al., 2009; Vaidyanathan and Chen, 1995; Zhang and Makur, 2009) and perfect deconvolution (Inouye and Tanebe, 2000; Tuncer, 2004), also in the problem of image recovery (Castella and Pesquet, 2004; Harikumar and Bresler, 1999a; $1999 b)$. On the other hand, the problem of perfect input reconstruction that has been solved employing the statespace approach (Edelmayer et al., 2004) could as well be tackled and resolved on the basis of the rational matrix system description.

It is striking how right/left inverses of polynomial matrices can contribute to the 'perfectualization' of signal transmission/reconstruction in various types of (generalized) MIMO channels. Although the perfect 'behavior' of MIMO channels is rather a theoretical, limiting property hardly attainable in the real, disturbance-corrupted world, inverse polynomial matrices can still be the foundation for application-oriented solutions. One example is robust MVC taking advantage of the ability of selecting inverse polynomial matrices with arbitrary poles and controlled zeros (Hunek, 2008; 2009b; Hunek et al., 2010). 


\section{Discussion and open problems}

One may argue that we should not use the term 'minimumnorm' $T$-inverse since, when solving the MVC equation (10) (or (11)) presented in the $z$-domain form

$$
B(z) U(z)=z^{d} \underline{Y}(z)
$$

with $U(z)$ and $\underline{Y}(z)$ corresponding to $u(t)$ and $\underline{y}(t)$, respectively, the actual minimum-norm solution would involve $B_{*}^{\mathrm{R}}(z)=B^{*}(z)\left[B(z) B^{*}(z)\right]^{-1}$, with $\left\|U_{*}(z)\right\|^{2}=$ $U^{*}(z) U(z)$, where the asterisk denotes the conjugate transpose. In fact, $\left\|U_{*}(z)\right\| \leq\left\|U_{0}(z)\right\|$, where $\left\|U_{0}(z)\right\|$ is the norm involving the $T$-inverse, that is, the regular rather than conjugate transpose. Now, the term 'minimumnorm' solution/inverse should be reserved for the conjugate transpose-based norm. Similar should hold for the least-squares solution/inverse, involving $B_{*}^{\mathrm{L}}(z)=$ $\left[B^{*}(z) B(z)\right]^{-1} B^{*}(z)$. We will show that such an argument is not true and the $z$-domain approach is not proper for the solution of the inverse-model control problem considered.

Firstly, the MVC Equation (10) (or (11)) is the time-domain one. That means that a norm of a timedomain solver to this equation must use real-valued features, that is, regular rather than conjugate transposes. Thus, the minimum-norm or least-squares solutions/inverses must end up with the $T$-inverses, involving $\underline{B}_{0}^{\mathrm{R}}\left(q^{-1}\right)=\underline{B}^{\mathrm{T}}\left(q^{-1}\right)\left[\underline{B}\left(q^{-1}\right) \underline{B}^{\mathrm{T}}\left(q^{-1}\right)\right]^{-1}$ (or $\left.\underline{B}_{0}^{\mathrm{L}}\left(q^{-1}\right)=\left[\underline{B}^{\mathrm{T}}\left(q^{-1}\right) \underline{B}\left(q^{-1}\right)\right]^{-1} \underline{B}^{\mathrm{T}}\left(q^{-1}\right)\right)$, with the minimal norm (or minimal energy)

$$
\|u(t)\|=\sqrt{\sum_{t=0}^{\infty} u^{\mathrm{T}}(t) u(t)}
$$

provided. (Note that the finite-time norm is practically employed, marking the steady-state conditions.) Therefore, we use the term 'minimum-norm/least-squares $T$-inverse' to additionally stress that the regular transpose is involved for the time-domain signals considered.

Secondly, the $z$-domain solution to Eqn. (26) would not take us any further, in terms of the inability to transform it to the time-domain solution. The reason is obvious: the $z$-domain solution would involve $\bar{z}=\operatorname{Re} z-i \operatorname{Im} z$, in general.

To additionally exemplify the idea of the minimumnorm $T$-inverse/solution, consider the nonsquare full normal rank MFD system specified in Appendix E. Choosing $\beta(z)=\left[\begin{array}{ll}I_{n_{y}} & 0_{n_{y} \times\left(n_{u}-n_{y}\right)}\end{array}\right]$, we end up with the (dimension reducing or squaring down) $\sigma$-inverse

$$
B^{\mathrm{R}}(z)=\left[\begin{array}{c}
\Psi_{n_{y} \times n_{y}} \\
0_{\left(n_{u}-n_{y}\right) \times n_{y}}
\end{array}\right],
$$

with $\Psi=\Psi(z)$ being a rational matrix, which implies that the associated system under MVC would be a squared-down $\left(n_{y} \times n_{y}\right)$ control one. It might seem that in such a case the time-domain norm of the MV/pefect control signal could be lower than that for the minimumnorm $T$-solution. This cannot be true: the minimumnorm $T$-solution is really the minimum norm one, that is, $\left\|u_{0}(t)\right\|<\left\|u_{\sigma}(t)\right\|$, where $u_{0}(t)$ and $u_{\sigma}(t)$ are the $\mathrm{MV} /$ perfect control signals involving the $T$-inverse and the specific $\sigma$-inverse of $\underline{B}\left(q^{-1}\right)$, respectively. The same can be verified in yet another interesting example with the $10 \times 1$ MISO system specified in Appendix F and

$$
\beta(z)=\left[\begin{array}{lllllll}
0 & \ldots & 0 & 1 & 0 & \ldots & 0
\end{array}\right]
$$

producing the $\sigma$-inverse

$$
B^{\mathrm{R}}(z)=\left[\begin{array}{lllllll}
0 & \ldots & 0 & \underset{i}{\Psi} & 0 & \ldots & 0
\end{array}\right]^{\mathrm{T}},
$$

that is, with the only nonzero entries at the $i$-th position, $i=1, \ldots, 10$, and $\Psi=\Psi(z)$ being a rational matrix. In fact, in all the squared-down SISO control systems obtained we have $\left\|u_{0}(t)\right\|<\left\|u_{\sigma}^{i}(t)\right\|$ for each $i=1, \ldots, 10$.

One may also argue that the notions 'minimum-norm right inverse' and 'least-squares left inverse' are justified for real-valued parameter matrices $B^{\mathrm{R}}=B^{\mathrm{T}}\left(B B^{\mathrm{T}}\right)^{-1}$ and $B^{\mathrm{L}}=\left(B^{\mathrm{T}} B\right)^{-1} B^{\mathrm{T}}$, respectively, so that transferring them to polynomial matrices (and rational matrices) introduces doubts and reservations. Although the transferring problem itself is indeed an open issue, we wish to stress once more that our solution is the time-domain one and there is no such transferring needed in our case at all. An attempt to find the minimum-norm solution in the Frobenius norm-involved polynomial matrix case has been presented by Kwan and Kok (2006), but it can by no means be applied to our time-domain formulated inverse problem.

It is worth emphasizing once more that, although our solution to the inverse-model control problem is timedomain, all the introduced polynomial matrix inverses can be used both in the time domain-valued $\underline{B}^{\mathrm{R}}\left(q^{-1}\right)$ form and the complex operator-associated version $B^{\mathrm{R}}(z)$. In other words, in our control synthesis problem we can operate either with $\underline{B}^{\mathrm{R}}\left(q^{-1}\right)$ or $\underline{B}^{\mathrm{R}}\left(z^{-1}\right)$ or $B^{\mathrm{R}}(z)$, although with the associated time-domain norms involving regular transposes. Therefore, we have exchangeably operated with various arguments of polynomial/rational matrices.

The computation of right/left inverses for nonsquare polynomial matrices is a rather unexplored research area, so the new results obtained in the paper are accompanied with a series of open problems. In addition to a general open issue of possible transferring of minimumnorm/least-squares inverses of parameter matrices to the polynomial matrix case (which is of no interest in our case), there have been a number of detailed open problems scattered throughout the paper and specified below. Since $\tau$-inverses have appeared not to be of practical interest, we have skipped over the open issue of formal defining 
of the inverses, which might deserve a research effort, at least from a theoretical point of view. Likewise, a theoretical analysis of unfavorable properties of $\tau$-inverses, in terms of the appearance of stable/unstable poles, might be welcome.

The new $\sigma$-inverses are definitely worth further research effort. Firstly, the difficult problem of proving Conjecture 1 presents a research challenge. Secondly, a general method for the selection of $\beta\left(q^{-1}\right)$ to provide a stable $\sigma$-inverse is awaiting its developer. Similarly, we lack a general methodology for designing $S$-inverses with arbitrary zeros. Also, it might be interesting to analyze properties of $\sigma$-inverses when $\underline{\beta}\left(q^{-1}\right)$ is not of full normal rank, even though in those cases $\underline{B}\left(q^{-1}\right) \underline{B}^{\mathrm{R}}\left(q^{-1}\right) \neq I_{n_{y}}$, in general. Last but not least, it is of interest to seek a relation between the unique finite/infinite zero structure of the Moore-Penrose inverse of a nonsquare polynomial matrix and a finite/infinite zero structure of that matrix, the open issue brought to the authors' attention by one of the anonymous reviewers. The above open problems are the subject of our current research works, in addition to seeking further application areas for nonsquare inverse polynomial matrices.

\section{Conclusions}

In this paper, new analytical expressions for various right/left inverses of full normal rank nonsquare polynomial matrices have been presented. The new inverses have been shown to originate from the minimum variance control strategy, the application that has stimulated research interest in this strictly mathematical field. In addition to the well-known but renamed $T$-inverses, three new classes of the inverses have been introduced, that is, $\tau$-inverses, $\sigma$-inverses and $S$-inverses. Also, the extreme points and extreme directions method has been adopted to the inverse polynomial matrix solution, but the approach has not been found promising here.

A number of the most algorithmically complicated $\tau$ inverses is finite so that their application is limited, even though nice, combinatorics-related, algorithmic and technical results have been achieved. A number of more general, algorithmically simple $\sigma$-inverses is infinite but, so far, they suffer from the lack of a general methodology for designing stable-pole polynomial matrix inverses, a crucial requirement in control-related applications. This disadvantage of $\sigma$-inverses is removed in the most general and algorithmically simple $S$-inverses, employing the Smith factorization approach.

In addition to 'natural' applications of the introduced polynomial matrix inverses in inverse-model control, in particular robust minimum variance control, possible applications in a variety of communication/vision 'perfectualizing' tasks have been indicated, with all of them still waiting for an extension of the up-to-date exploited minimum-norm/least-squares polynomial matrix inverses.

\section{Acknowledgment}

Invaluable comments from the anonymous reviewers are gratefully acknowledged.

\section{References}

Bańka, S. and Dworak, P. (2006). Efficient algorithm for designing multipurpose control systems for invertible and right-invertible MIMO LTI plants, Bulletin of the Polish Academy of Sciences: Technical Sciences 54(4): 429-436.

Bańka, S. and Dworak, P. (2007). On decoupling of LTI MIMO systems with guaranteed stability, Measurement Automation and Monitoring 53(6): 46-51.

Ben-Israel, A. and Greville, T.N.E. (2003). Generalized Inverses, Theory and Applications, 2nd Edn., Springer-Verlag, New York, NY.

Bernardini, R. and Rinaldo, R. (2006). Oversampled filter banks from extended perfect reconstruction filter banks, IEEE Transactions on Signal Processing 54(7): 26252635, DOI: 10.1109/TSP.2006.874811.

Boche, H. and Pohl, V. (2006). MIMO-ISI channel equalization-Which price we have to pay for causality, Proceedings of the 14th European Signal Processing Conference (EUSIPCO'2006), Florence, Italy, (on CD-ROM).

Bose, N.K. and Mitra, S.K. (1978). Generalized inverse of polynomial matrices, IEEE Transactions on Automatic Control 23(3): 491-493.

Callier, F.M. and Kraffer, F. (2005). Proper feedback compensators for a strictly proper plant by polynomial equations, International Journal of Applied Mathematics and Computer Science 15(4): 493-507.

Castella, M. and Pesquet, J.-C. (2004). An iterative blind source separation method for convolutive mixtures of images, in C.G. Puntonet and A. Prieto (Eds.), Independent Component Analysis and Blind Signal Separation, Lecture Notes in Computer Science, Vol. 3195, Springer-Verlag, Heidelberg/Berlin, pp. 922-929.

Chen, J. and Hara, S. (2001). Tracking performance with finite input energy, in S.O.R. Moheimani (Ed.), Perspectives in Robust Control, Lecture Notes in Control and Information Sciences, Vol. 268, Springer-Verlag, Heidelberg/Berlin, Chapter 4, pp. 41-55.

Davison, E. (1983). Some properties of minimum phase systems and 'squared-down' systems, IEEE Transactions on Automatic Control 28(2): 221-222.

Desoer, C.A. and Schulman, J.D. (1974). Zeros and poles of matrix transfer functions and their dynamical interpretation, IEEE Transactions on Circuits and Systems 21(1): 3-8.

Edelmayer, A., Bokor, J., Szabó, Z. and Szigeti, F. (2004). Input reconstruction by means of system inversion: A geometric approach to fault detection and isolation in nonlinear systems, International Journal of Applied Mathematics and Computer Science 14(2): 189-199. 
Ferreira, P.M.G. (1988). Some results on system equivalence, International Journal of Control 48(5): 2033-2042, DOI: 10.1080/00207178808906303.

Fornasini, E. and Pinto, R. (2004). Matrix fraction descriptions in convolutional coding, Linear Algebra and Its Applications 392: 119-158, DOI: 10.1016/j.laa.2004.06.007.

Forney, Jr., G.D. (1991). Algebraic structure of convolutional codes, and algebraic system theory, in A.C. Antoulas (Ed.), Mathematical System Theory: The Influence of R.E. Kalman, Springer-Verlag, Heidelberg/Berlin, pp. 527-557.

Gan, L. and Ling, C. (2008). Computation of the para-pseudoinverse for oversampled filter banks: Forward and backward Greville formulas, IEEE Transactions on Signal Processing 56(12): 5851-5860, DOI: 10.1109/TSP.2008.2005086.

Gluesing-Luerssen, H., Rosenthal, J. and Smarandache, R. (2006). Strongly-MDS convolutional codes, IEEE Transactions on Information Theory 52(2): 584-598, DOI: 10.1109/TIT.2005.862100.

Guo, Y. and Levy, B.C. (2004). Design of FIR precoders and equalizers for broadband MIMO wireless channels with power constraints, EURASIP Journal on Wireless Communications and Networking 2004(2): 344-356, DOI: 10.1155/S1687147204406185.

Harikumar, G. and Bresler, Y. (1999a). Exact image deconvolution from multiple FIR blurs, IEEE Transactions on Image Processing 8(6): 846-862, DOI: 10.1109/83.766861.

Harikumar, G. and Bresler, Y. (1999b). Perfect blind restoration of images blurred by multiple filters: Theory and efficient algorithms, IEEE Transactions on Image Processing 8(2): 202-219, DOI: 10.1109/83.743855.

Hautus, M.L.J. and Heymann, M. (1983). Linear feedback decoupling-Transfer function analysis, IEEE Transactions on Automatic Control 28(8): 823-832.

Henrion, D. (1998). Reliable Algorithms for Polynomial Matrices, Ph.D. thesis, Institute of Information Theory and Automation, Czech Academy of Sciences, Prague.

Hunek, W.P. (2008). Towards robust minimum variance control of nonsquare LTI MIMO systems, Archives of Control Sciences 18(1): 59-71.

Hunek, W.P. (2009a). A new general class of MVC-related inverses of nonsquare polynomial matrices based on the Smith factorization, Proceedings of the 14th IEEE IFAC International Conference on Methods and Models in Automation and Robotics (MMAR'2009), Międzyzdroje, Poland, (on CD-ROM).

Hunek, W.P. (2009b). Towards robust pole-free designs of minimum variance control for LTI MIMO systems, in S. Pennacchio (Ed.), Recent Advances in Control Systems, Robotics and Automation, 3rd Edn., International Society for Advanced Research, Palermo, pp. 168-174.

Hunek, W.P. and Latawiec, K.J. (2009). Minimum variance control of discrete-time and continuous-time LTI MIMO systems-A new unified framework, Control and Cybernetics 38(3): 609-624.
Hunek, W.P., Latawiec, K.J., Łukaniszyn, M. and Gawdzik, A. (2010). Constrained minimum variance control of nonsquare LTI MIMO systems, 15th IEEE International Conference on Methods and Models in Automation and Robotics (MMAR'2010), Międzyzdroje, Poland, pp. 365-370.

Hunek, W.P., Latawiec, K.J. and Stanisławski, R. (2007). New results in control zeros vs. transmission zeros for LTI MIMO systems, Proceedings of the 13th IEEE IFAC International Conference on Methods and Models in Automation and Robotics (MMAR'2007), Szczecin, Poland, pp. 149153.

Inouye, Y. and Tanebe, K. (2000). Super-exponential algorithms for multichannel blind deconvolution, IEEE Transactions on Signal Processing 48(3): 881-888, DOI: $10.1109 / 78.824685$.

Johannesson, R., Wan, Z.-X. and Wittenmark, E. (1998). Some structural properties of convolutional codes over rings, $I E$ EE Transactions on Information Theory 44(2): 839-845, DOI: $10.1109 / 18.661532$.

Johannesson, R. and Zigangirov, K.S. (1999). Fundamentals of Convolutional Coding, IEEE Press, New York, NY.

Kaczorek, T. (2005). Extension of the Cayley-Hamilton theorem for right and left inverses of polynomial matrices and rational matrices, Electrical Review 81(11): 66-71.

Kaczorek, T., Dzieliński, A., Dąbrowski, W. and Łopatka, R. (2009). Fundamentals of Control Theory, 3rd Edn., Wydawnictwa Naukowo-Techniczne, Warsaw, (in Polish).

Kaczorek, T. and Łopatka, R. (2000). Existence and computation of the set of positive solutions to polynomial matrix equations, International Journal of Applied Mathematics and Computer Science 10(2): 309-320.

Kailath, T. (1980). Linear Systems, Prentice Hall, Englewood Cliffs, NJ.

Karampetakis, N.P. and Tzekis, P. (2001). On the computation of the generalized inverse of a polynomial matrix, IMA Journal of Mathematical Control and Information 18(1): 8397, DOI: 10.1093/imamci/18.1.83.

Kim, S. and Park, Y. (2004). A direct design method of inverse filters for multichannel 3D sound rendering, Journal of Sound and Vibration 278(4-5): 1196-1204, DOI: 10.1016/j.jsv.2003.12.014.

Kon'kova, T.Y. and Kublanovskaya, V.N. (1996). Inversion of polynomial and rational matrices, Journal of Mathematical Sciences 79(3): 1093-1100, DOI: 10.1007/BF02366129.

Kwan, M.W. and Kok, C.W. (2006). Iterative joint optimization of minimal transmit redundancy FIR zero-forcing precoder-equalizer system for MIMO-ISI channel, IEEE Transactions on Signal Processing 54(1): 204-213, DOI: 10.1109/TSP.2005.861059.

Latawiec, K.J. (1998). Contributions to Advanced Control and Estimation for Linear Discrete-Time MIMO Systems, Opole University of Technology Press, Opole.

Latawiec, K.J. (2004). The Power of Inverse Systems in Linear and Nonlinear Modeling and Control, Opole University of Technology Press, Opole. 
Latawiec, K.J. (2005). Control zeros and maximum-accuracy/ maximum-speed control of LTI MIMO discrete-time systems, Control and Cybernetics 34(2): 453-475.

Latawiec, K.J., Bańka, S. and Tokarzewski, J. (2000). Control zeros and nonminimum phase LTI MIMO systems, Annual Reviews in Control 24(1): 105-112. DOI: 10.1016/S13675788(00)90021-X.

Latawiec, K.J., Hunek, W.P. and Łukaniszyn, M. (2004). A new type of control zeros for LTI MIMO systems, Proceedings of the 10th IEEE International Conference on Methods and Models in Automation and Robotics (MMAR'2004), Międzyzdroje, Poland, pp. 251-256.

Latawiec, K.J., Hunek, W.P. and Łukaniszyn, M. (2005). New optimal solvers of MVC-related linear matrix polynomial equations, Proceedings of the 11th IEEE International Conference on Methods and Models in Automation and Robotics (MMAR'2005), Międzyzdroje, Poland, pp. 333-338.

Law, K.L., Fossum, R.M. and Do, M.N. (2009). Generic invertibility of multidimensional FIR filter banks and MIMO systems, IEEE Transactions on Signal Processing 57(11): 4282-4291, DOI: 10.1109/TSP.2009.2025826.

Lin, S. and Costello, D.J. (2004). Error Control Coding, 2nd Edn., Prentice Hall, Upper Saddle River, NJ.

Lu, P., Li, S., Zou, Y. and Luo, X. (2005). Blind recognition of punctured convolutional codes, Science in China, Series F: Information Sciences 48(4): 484-498, DOI: 10.1360/03yf0480.

Moon, T.K. (2005). Error Correction Coding: Mathematical Methods and Algorithms, John Wiley \& Sons, Hoboken, NJ.

Quadrat, A. (2004). On a general structure of the stabilizing controllers based on stable range, SIAM Journal on Control and Optimization 42(6): 2264-2285, DOI: $10.1137 /$ S0363012902408277.

Quevedo, D.E., Bölcskei, H. and Goodwin, G.C. (2009). Quantization of filter bank frame expansions through moving horizon optimization, IEEE Transactions on Signal Processing 57(2): 503-515, DOI: 10.1109/TSP.2008.2008259.

Sontag, E.D. (1980). On generalized inverses of polynomial and other matrices, IEEE Transactions on Automatic Control 25(3): 514-517.

Stanimirović, P.S. (2003). A finite algorithm for generalized inverses of polynomial and rational matrices, Applied Mathematics and Computation 144(2-3): 199-214, DOI: 10.1016/S0096-3003(02)00401-0.

Stanimirović, P.S. and Petković, M.D. (2006). Computing generalized inverse of polynomial matrices by interpolation, Applied Mathematics and Computation 172(1): 508-523, DOI: 10.1016/j.amc.2005.02.031.

Tidestav, C., Sternad, M. and Ahlen, A. (1999). Reuse within a cell-interference rejection or multiuser detection?, $I E$ EE Transactions on Communications 47(10): 1511-1522, DOI: $10.1109 / 26.795820$.

Tokarzewski, J. (2002). Zeros in Linear Systems: A Geometric Approach, Warsaw University of Technology Press, Warsaw.
Tokarzewski, J. (2004). A note on some characterization of invariant zeros in singular systems and algebraic criteria of nondegeneracy, International Journal of Applied Mathematics and Computer Science 14(2): 149-159.

Trentelman, H.L., Stoorvogel, A.A. and Hautus, M. (2001) Control Theory for Linear Systems, Communications and Control Engineering, Springer-Verlag, New York, NY.

Tuncer, T.E. (2004). Deconvolution and preequalization with best delay LS inverse filters, Signal Processing 84(11): 2207-2219, DOI: 10.1016/j.sigpro.2004.06.023.

Vaidyanathan, P. P. and Chen, T. (1995). Role of anticausal inverses in multirate filter-banks-Part I: System-theoretic fundamentals, IEEE Transactions on Signal Processing 43(5): 1090-1102, DOI: 10.1109/78.382395.

Vardulakis, A.I.G. (1991). Linear Multivariable Control: Algebraic Analysis and Synthesis Methods, 1st Edn., John Wiley \& Sons, Chichester.

Varga, A. (2001). Computing generalized inverse systems using matrix pencil methods, International Journal of Applied Mathematics and Computer Science 11(5): 1055-1068.

Vologiannidis, S. and Karampetakis, N.P. (2004). Inverses of multivariable polynomial matrices by discrete Fourier transforms, Multidimensional Systems and Signal Processing 15(4): 341-361, DOI: 10.1023/B:MULT.0000037345.60574.d4.

Wahls, S. and Boche, H. (2007). Stable and causal LTI-precoders and equalizers for MIMO-ISI channels with optimal robustness properties, Proceedings of the International ITG/IEEE Workshop on Smart Antennas (WSA'2007), Vienna, Austria, (on CD-ROM).

Wahls, S., Boche, H. and Pohl, V. (2009). Zero-forcing precoding for frequency selective MIMO channels with $H^{\infty}$ criterion and causality constraint, Signal Processing 89(9): 1754-1761, DOI: 10.1016/j.sigpro.2009.03.010.

Williams, T. and Antsaklis, P.J. (1996). Decoupling, in W.S. Levine (Ed.), The Control Handbook, Electrical Engineering Handbook, CRC Press/IEEE Press, Boca Raton, FL, Chapter 50, pp. $745-804$.

Xia, X.-G., Su, W. and Liu, H. (2001). Filterbank precoders for blind equalization: Polynomial ambiguity resistant precoders (PARP), IEEE Transactions on Circuits and SystemsI: Fundamental Theory and Applications 48(2): 193-209, DOI: $10.1109 / 81.904884$.

Zhang, L. and Makur, A. (2009). Multidimensional perfect reconstruction filter banks: An approach of algebraic geometry, Multidimensional Systems and Signal Processing 20(1): 3-24, DOI: 10.1007/s11045-008-0060-5.

Zhang, S.Y. (1989). Generalized proper inverse of polynomial matrices and the existence of infinite decoupling zeros, IEEE Transaction on Automatic Control 34(7): 743-745, DOI: $10.1109 / 9.29403$. 


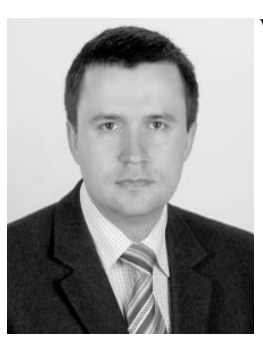

Wojciech P. Hunek received his M.Sc. and Ph.D. degrees in electrical engineering from the Faculty of Electrical, Control and Computer Engineering, Opole University of Technology, in 1999 and 2003, respectively. He is an assistant professor at the Institute of Control and Computer Engineering. He has authored or co-authored some 50 papers, concerning mainly modern ideas in multivariable control theory.

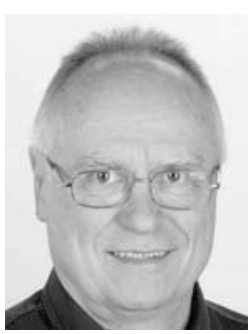

Krzysztof J. Latawiec received his habilitation degree in control and robotics from the $\mathrm{AGH}$ University of Science and Technology in Cracow, Poland, in 1999. Currently he is a Professor of control and robotics and the head of the Control and Electronics Group at the Faculty of Electrical, Control and Computer Engineering, Opole University of Technology, Poland. His research interests concentrate on system identificacontrol (also in networks), and fractional systems.

\section{Appendix A}

$\operatorname{Expanding} \underline{B}^{\mathrm{R} 5}$ for $\underline{\beta}\left(q^{-1}\right)=\underline{b}_{0}+\underline{b}_{1} q^{-1}$ and

$$
\left(\underline{b}_{0}+\underline{b}_{1} q^{-1}\right)^{\mathrm{R}}=\left[I+\left(\underline{b}_{0}\right)^{\mathrm{R}} \underline{b}_{1} q^{-1}\right]^{-1}\left(\underline{b}_{0}\right)^{\mathrm{R}},
$$

we arrive at

$$
\begin{aligned}
\underline{B}^{\mathrm{R} 8}\left(q^{-1}\right)= & \left\{I+\left\{\left[I+\left(\underline{b}_{0}\right)^{\mathrm{R}} \underline{b}_{1} q^{-1}\right]^{-1}\left(\underline{b}_{0}\right)^{\mathrm{R}}\right\}\right. \\
& \left.\times\left(\underline{b}_{2} q^{-2}\right)\right\}^{-1}\left\{\left[I+\left(\underline{b}_{0}\right)^{\mathrm{R}} \underline{b}_{1} q^{-1}\right]^{-1}\left(\underline{b}_{0}\right)^{\mathrm{R}}\right\},
\end{aligned}
$$

while for

$$
\left(\underline{b}_{0}+\underline{b}_{1} q^{-1}\right)^{\mathrm{R}}=\left[I+\left(\underline{b}_{1} q^{-1}\right)^{\mathrm{R}} \underline{b}_{0}\right]^{-1}\left(\underline{b}_{1} q^{-1}\right)^{\mathrm{R}}
$$

we have

$$
\begin{aligned}
\underline{B}^{\mathrm{R} 9}\left(q^{-1}\right)= & \left\{I+\left\{\left[I+\left(\underline{b}_{1} q^{-1}\right)^{\mathrm{R}} \underline{b}_{0}\right]^{-1}\left(\underline{b}_{1} q^{-1}\right)^{\mathrm{R}}\right\}\right. \\
& \left.\times\left(\underline{b}_{2} q^{-2}\right)\right\}^{-1}\left\{\left[I+\left(\underline{b}_{1} q^{-1}\right)^{\mathrm{R}} \underline{b}_{0}\right]^{-1}\right. \\
& \left.\times\left(\underline{b}_{1} q^{-1}\right)^{\mathrm{R}}\right\} .
\end{aligned}
$$

By the same token, expanding $\underline{B}^{\mathrm{R} 6}$ for $\underline{\beta}\left(q^{-1}\right)=$ $\underline{b}_{0}+\underline{b}_{2} q^{-2}$ with

$$
\left(\underline{b}_{0}+\underline{b}_{2} q^{-2}\right)^{\mathrm{R}}=\left[I+\left(\underline{b}_{0}\right)^{\mathrm{R}} \underline{b}_{2} q^{-2}\right]^{-1}\left(\underline{b}_{0}\right)^{\mathrm{R}}
$$

and

$$
\left(\underline{b}_{0}+\underline{b}_{2} q^{-2}\right)^{\mathrm{R}}=\left[I+\left(\underline{b}_{2} q^{-2}\right)^{\mathrm{R}} \underline{b}_{0}\right]^{-1}\left(\underline{b}_{2} q^{-2}\right)^{\mathrm{R}}
$$

we obtain

$$
\begin{aligned}
\underline{B}^{\mathrm{R} 10} & \left(q^{-1}\right) \\
= & \left\{I+\left\{\left[I+\left(\underline{b}_{0}\right)^{\mathrm{R}} \underline{b}_{2} q^{-2}\right]^{-1}\left(\underline{b}_{0}\right)^{\mathrm{R}}\right\}\right. \\
& \left.\times\left(\underline{b}_{1} q^{-1}\right)\right\}^{-1}\left\{\left[I+\left(\underline{b}_{0}\right)^{\mathrm{R}} \underline{b}_{2} q^{-2}\right]^{-1}\left(\underline{b}_{0}\right)^{\mathrm{R}}\right\}
\end{aligned}
$$

and

$$
\begin{aligned}
\underline{B}^{\mathrm{R} 11} & \left(q^{-1}\right) \\
= & \left\{I+\left\{\left[I+\left(\underline{b}_{2} q^{-2}\right)^{\mathrm{R}} \underline{b}_{0}\right]^{-1}\right.\right. \\
& \left.\left.\times\left(\underline{b}_{2} q^{-2}\right)^{\mathrm{R}}\right\}\left(\underline{b}_{1} q^{-1}\right)\right\}^{-1} \\
& \times\left\{\left[I+\left(\underline{b}_{2} q^{-2}\right)^{\mathrm{R}} \underline{b}_{0}\right]^{-1}\left(\underline{b}_{2} q^{-2}\right)^{\mathrm{R}}\right\},
\end{aligned}
$$

respectively. with

Finally, expanding $\underline{B}^{\mathrm{R} 7}$ for $\underline{\beta}\left(q^{-1}\right)=\underline{b}_{1} q^{-1}+\underline{b}_{2} q^{-2}$

$$
\left(\underline{b}_{1} q^{-1}+\underline{b}_{2} q^{-2}\right)^{\mathrm{R}}=\left[I+\left(\underline{b}_{1} q^{-1}\right)^{\mathrm{R}} \underline{b}_{2} q^{-2}\right]^{-1}\left(\underline{b}_{1} q^{-1}\right)^{\mathrm{R}}
$$

and

$$
\left(\underline{b}_{1} q^{-1}+\underline{b}_{2} q^{-2}\right)^{\mathrm{R}}=\left[I+\left(\underline{b}_{2} q^{-2}\right)^{\mathrm{R}} \underline{b}_{1} q^{-1}\right]^{-1}\left(\underline{b}_{2} q^{-2}\right)^{\mathrm{R}}
$$

we have

$$
\begin{aligned}
\underline{B}^{\mathrm{R} 12} & \left(q^{-1}\right) \\
= & \left\{I+\left\{\left[I+\left(\underline{b}_{1} q^{-1}\right)^{\mathrm{R}} \underline{b}_{2} q^{-2}\right]^{-1}\left(\underline{b}_{1} q^{-1}\right)^{\mathrm{R}}\right\}\right. \\
& \left.\times\left(\underline{b}_{0}\right)\right\}^{-1}\left\{\left[I+\left(\underline{b}_{1} q^{-1}\right)^{\mathrm{R}} \underline{b}_{2} q^{-2}\right]^{-1}\left(\underline{b}_{1} q^{-1}\right)^{\mathrm{R}}\right\}
\end{aligned}
$$

and

$$
\begin{aligned}
\underline{B}^{\mathrm{R} 13}\left(q^{-1}\right) & \\
= & \left\{I+\left\{\left[I+\left(\underline{b}_{2} q^{-2}\right)^{\mathrm{R}} \underline{b}_{1} q^{-1}\right]^{-1}\left(\underline{b}_{2} q^{-2}\right)^{\mathrm{R}}\right\}\right. \\
& \left.\times\left(\underline{b}_{0}\right)\right\}^{-1}\left\{\left[I+\left(\underline{b}_{2} q^{-2}\right)^{\mathrm{R}} \underline{b}_{1} q^{-1}\right]^{-1}\left(\underline{b}_{2} q^{-2}\right)^{\mathrm{R}}\right\},
\end{aligned}
$$

respectively.

Note that all the above identity matrices shall be read as $I_{n_{u}}$.

\section{Appendix B}

Proof of Theorem 1. Table 1 gives the numbers of $\tau$ inverses calculated according to Algorithm 1 at each step $k=1, \ldots, m$ and for each set $i=1, \ldots, m$ of the inverses. Table 2 is a sample of Table 1 for $m=4$. We use the standard notation for $C_{k}^{i}=k ! /[i !(k-i) !]$ as the number of $i$-combinations without repetition from a $k$-element set.

It is essential that we calculate the total number $S$ of $\tau$-inverses as a sum of all the subsums counted by columns

$$
S=1+\sum_{i=1}^{m} C_{m+1}^{i} \sum_{k=1}^{i} F_{k, i},
$$

with $C_{m+1}^{m+1}=1$ being the number of the $T$-inverse. The constructions of the numbers of inverses for each set $i=$ $1, \ldots, m$ allows calculating the factor

$$
\sum_{k=1}^{i} F_{k, i}=N_{i-1}, \quad i=1, \ldots, m,
$$

in a recurrent way. Specifically, for the column $i=2$ we have $F_{12}+F_{22}=N_{1}=1+C_{2}^{1} N_{0}$, where $N_{0}=1$, and for the column $i=3$ there is $F_{13}+F_{23}+F_{33}=N_{2}=$ $1+C_{3}^{1} N_{0}+C_{3}^{2} N_{1}$. 


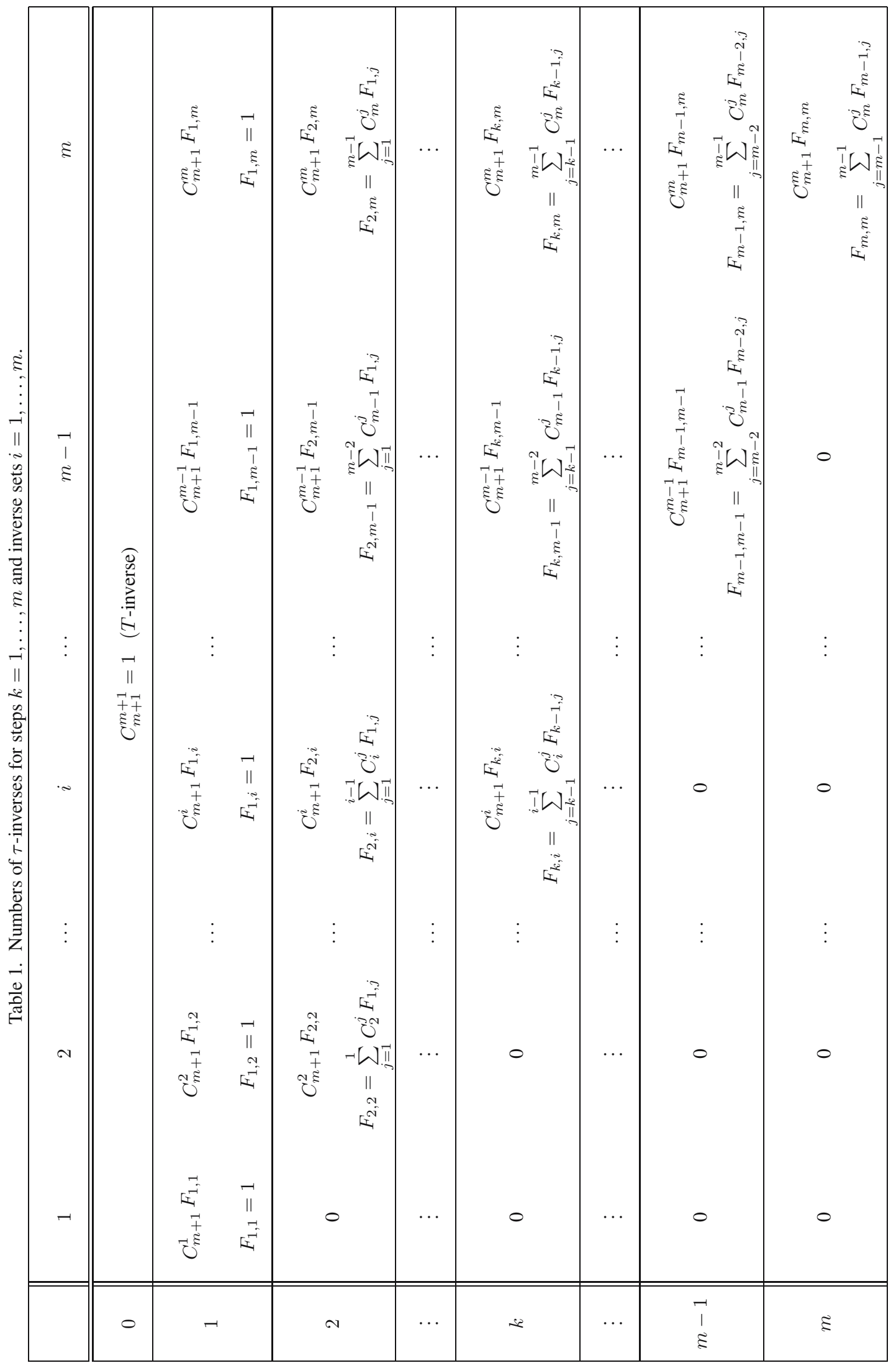


Table 2. Example of Table 1 for $m=4$.

\begin{tabular}{|c|c|c|c|c|}
\hline & 1 & 2 & 3 & 4 \\
\hline 0 & \multicolumn{4}{|c|}{$C_{5}^{5}$} \\
\hline 1 & $C_{5}^{1}$ & $C_{5}^{2}$ & $C_{5}^{3}$ & $C_{5}^{4}$ \\
\hline 2 & 0 & $C_{5}^{2} C_{2}^{1}$ & $C_{5}^{3}\left(C_{3}^{1}+C_{3}^{2}\right)$ & $C_{5}^{4}\left(C_{4}^{1}+C_{4}^{2}+C_{4}^{3}\right)$ \\
\hline 3 & 0 & 0 & $C_{5}^{3} C_{3}^{2} C_{2}^{1}$ & $C_{5}^{4} C_{4}^{2} C_{2}^{1}+C_{5}^{4} C_{4}^{3}\left(C_{3}^{1}+C_{3}^{2}\right)$ \\
\hline 4 & 0 & 0 & 0 & $C_{5}^{4} C_{4}^{3} C_{3}^{2} C_{2}^{1}$ \\
\hline
\end{tabular}

The general recurrence can be easily shown to be

$$
N_{i}=1+\sum_{j=1}^{i} C_{i+1}^{j} N_{j-1}, \quad i=1, \ldots, m-1 .
$$

The last recurrence is obviously included in Eqn. (27) with $S=N_{m}$. Expanding $C_{i+1}^{j}$ yields the final result (13).

\section{Appendix C}

Control zeros for $B(z)$ as in Example 1.

- unstable $B^{\mathrm{R}}(z): \quad 0.1, \quad 0.1063 \pm 0.4429 i$, $0.0190 \pm 1.8760 i$;

- stable $B^{\mathrm{R}}(z): 0.1,0.2715 \pm 0.5377 i, 0.0032 \pm$ $0.7353 i$.

\section{Appendix D}

Matrices $Z_{1}(w)$ and $Z_{2}(w)$ in Example 2 .

$$
\begin{aligned}
& Z_{1}(w)=\left[\begin{array}{cc}
2+2.4 w+0.2 w^{2}+w^{3} & 1.9+1.1 w-1.3 w^{2}+2.1 w^{3}+0.9 w^{4}+0.062 w^{5}+0.65 w^{6} \\
0.24-1.5 w+0.21 w^{2}-0.69 w^{3} & 0.25-1.7 w+w^{2}-0.61 w^{3}-0.54 w^{4}+0.17 w^{5}-0.43 w^{6} \\
-0.48+1.2 w+0.34 w^{2} & -0.82+1.6 w-0.3 w^{2}-0.57 w^{3}+0.75 w^{4}+0.22 w^{5}
\end{array}\right] \\
& Z_{2}(w)=\left[\begin{array}{cc}
1.8+0.87 w-2.8 w^{2}+1.2 w^{3}-1.3 w^{4} & \ldots \\
0.87-2.3 w+2.2 w^{2}-1.2 w^{3}+0.84 w^{4} & \ldots
\end{array}\right. \\
& -0.85+2.2 w-w^{2}-0.42 w^{3} \quad \ldots \\
& \ldots \quad 1.5-4 w-11 w^{2}+4.2 w^{3}-4.3 w^{4}-1.1 w^{5}+0.8 w^{6}-0.79 w^{7} \\
& \ldots \quad 2.4-5.1 w+8.3 w^{2}-3 w^{3}+2 w^{4}+0.97 w^{5}-0.8 w^{6}+0.53 w^{7} \\
& \ldots \quad-2.1+5.1 w-5.6 w^{2}-1.5 w^{3}+1.7 w^{4}-0.63 w^{5}-0.26 w^{6}
\end{aligned}
$$

with the squaring down $\sigma$-inverse

$$
B^{\mathrm{R}}(z)=\left[\begin{array}{c}
\Psi_{2 \times 2} \\
0_{2 \times 2}
\end{array}\right]
$$

$$
\beta(z)=\left[\begin{array}{ll}
I_{2} & 0_{2 \times 2}
\end{array}\right],
$$

providing a $2 \times 2 \mathrm{MV} /$ perfect control system with $\left\|u_{0}(t)\right\|<\left\|u_{\sigma}(t)\right\|$.

produced by
An example of a $4 \times 2$ MFD plant

\section{Appendix E}




\section{Appendix F}

An example of a $10 \times 1$ MFD plant

$$
\begin{array}{r}
G(z)=\frac{1}{z^{3}+0.5 z^{2}-0.5 z}\left[\begin{array}{rrrrr}
10 z^{2}+5 z+3 & z^{2}-0.5 z+0.3 & z^{2}+0.5 z+0.3 & 7 z^{2}+0.5 z-0.13 & \ldots \\
\ldots & 17 z^{2}+0.5 z+0.13 & z^{2}+0.5 z+0.1 & 1.1 z^{2}+0.52 z+0.11 & \ldots \\
\ldots & -2 z^{2}+0.5 z+0.4 & -2 z^{2}+z+0.4 & -12 z^{2}+5 z+4
\end{array}\right],
\end{array}
$$

with the squaring down $\sigma$-inverse

$$
B^{\mathrm{R}}(z)=\left[\begin{array}{lllllll}
0 & \ldots & 0 & \underset{i}{\Psi} & 0 & \ldots & 0
\end{array}\right]^{\mathrm{T}}
$$

produced by

$$
\beta(z)=\left[\begin{array}{lllllll}
0 & \ldots & 0 & 1 & 0 & \ldots & 0
\end{array}\right]
$$

providing SISO MV/perfect control systems with $\left\|u_{0}(t)\right\|<\left\|u_{\sigma}^{i}(t)\right\|$ for all $i=1, \ldots, 10$. For example, for $i=7$, we have the scalar $\Psi=\Psi(z)=1 /\left(1.1 z^{2}+0.52 z+0.11\right)$. 